Research Article

Open Access

Piotr Jaworski*

\title{
On Copula-Itô processes
}

https://doi.org/10.1515/demo-2019-0017

Received November 4, 2018; accepted September 30, 2019

Abstract: We study the dynamics of the family of copulas $\left\{C_{t}\right\}_{t \geq 0}$ of a pair of stochastic processes given by stochastic differential equations (SDE). We associate to it a parabolic partial differential equation (PDE). Having embedded the set of bivariate copulas in a dual of a Sobolev Hilbert space $H^{1}\left(\mathbb{R}^{2}\right)^{\star}$ we calculate the derivative with respect to $t$ and the * weak topology i.e. the tangent vector field to the image of the curve $t \rightarrow C_{t}$. Furthermore we show that the family $\left\{C_{t}\right\}_{t \geq 0}$ is an orbit of a strongly continuous semigroup of transformations and provide the infinitesimal generator of this semigroup.

Keywords: Copulas, copula processes, stochastic differential equations, parabolic partial differential equations, semigroups of transformations

MSC: 62H05, 60J60, 60H10, 46E35, 47H20, 58D07

\section{Introduction}

There is a vast literature dealing with the borderline of the copula theory and stochastic processes. There are two main objectives, temporal dependence and spatial dependence. The first one concerns the studies of dependencies over time: given a one-dimensional stochastic process $\left(X_{t}\right)_{t \in T}$ and $n$ times $t_{1}, \ldots, t_{n} \in T$ determine the copula of the random vector $\left(X_{t_{1}}, \ldots, X_{t_{n}}\right)$. The research in this direction was originated by seminal paper of Darsow, Nguyen and Olsen [7] and continued by many authors, amongst others Sempi [26], Bibbona et al. [2] and Schmitz [23]. The second one concerns the vector valued stochastic processes $X_{t}=$ $\left(X_{t}^{1}, \ldots, X_{t}^{n}\right), t \in T$. The goal is to describe the evolution of interdependencies between $X_{t}^{1}, \ldots, X_{t}^{n}$ in terms of copulas and copula processes. It is represented by papers by Sempi [25], Choe et al. [6] and Jaworski and Krzywda [12, 13].

This paper belongs to the second category. The work here is strongly influenced by the paper of Sempi ([25]) who was studying the possibility of coupling two Wiener processes by using a given copula, and the research on copulas of self-similar processes in [12, 13]. It generalized the results presented in [6].

Due to the celebrated Sklar's Theorem, one can split a study of a pair $(X, Y)$ of random variables into two parts. First one can deal with $X$ and $Y$ separately and then with the interdependence between them. In this paper we apply this methodology to the study of a pair of stochastic processes. We assume that dynamics of each process is described by a stochastic differential equation. Our objective is to describe the evolution of the interdependence. This is done in terms of a family of copulas $C_{t}$, linking the corresponding elements of the both processes. Having embedded the set of bivariate copulas $C^{2}$ into the dual to the Sobolev space $H^{1}\left(\mathbb{R}^{2}\right)$ we calculate the vector field tangent to the image of the family $\left(C_{t}\right)_{t \geq 0}$. Moreover, under some additional assumptions implying the existence and uniqueness of of the solutions of SDE, we show that there exists a continuous semigroup $\Lambda$ of transformations (endomorphisms) of the Cartesian product of nonnegative real numbers and the set of bivariate copulas $\mathbb{R}^{+} \times \mathcal{C}^{2}$

$$
\Lambda: \mathbb{R}^{+} \longrightarrow \operatorname{End}\left(\mathbb{R}^{+} \times \mathrm{C}^{2}\right)
$$

^Corresponding Author: Piotr Jaworski: Institute of Mathematics, University of Warsaw, Poland, Email:

P.Jaworski@mimuw.edu.pl 
such that the graph $\left(t, C_{t}\right)$ is an orbit of $\Lambda$. Having embedded the space of copulas into the dual to the Sobolev space $H^{1}\left(\mathbb{R}^{2}\right)$ we calculate the infinitesimal generator of $\Lambda$.

Structure of the paper:

First we shortly recall basics on copulas and the Sobolev space $H^{1}\left(\mathbb{R}^{2}\right)$ and its dual. Then we introduce the underlying stochastic processes $X_{t}^{1}$ and $X_{t}^{2}$ and the copula process $\left(C_{t}\right)_{t \geq 1}$. Next we show how to transform the stochastic differential equations of $X^{1}$ and $X^{2}$ to stochastic differential equations describing the processes of representers of the corresponding copulas $C_{t}$ (section 3). In section 4 we state our main results concerning the evolution of the family $\left(C_{t}\right)_{t \geq 0}$. We discuss the differentiability of $C_{t}$ with respect to $t$ and provide a parabolic differential equation for which the family $C_{t}$ serves as a weak solution. In the following (section 5 ) we construct the semigroup of transformations $\Lambda$ describing the dependence of the evolution of the families $C_{t}$ on initial values and discuss its properties. The proofs are relegated to section 6 .

\section{Notation}

We recall the following basic concepts.

A (bivariate) copula is a restriction to $[0,1]^{2}$ of a distribution function whose univariate margins are uniformly distributed on $[0,1]$. Specifically, $C:[0,1]^{2} \rightarrow[0,1]$ is a copula if it satisfies the following properties: (C1) $C(x, 0)=C(0, x)=0$ for every $x \in[0,1]$,

(C2) $C(x, 1)=C(1, x)=x$ for every $x \in[0,1]$,

(C3) $C$ is 2 -increasing, that is, the $C$-volume $V_{C}$ of any rectangle $\left[x_{1}, x_{2}\right] \times\left[y_{1}, y_{2}\right]$ of $[0,1]^{2}$ is positive, i.e.

$$
V_{C}\left(\left[x_{1}, x_{2}\right] \times\left[y_{1}, y_{2}\right]\right)=C\left(x_{1}, y_{1}\right)+C\left(x_{2}, y_{2}\right)-C\left(x_{1}, y_{2}\right)-C\left(x_{2}, y_{1}\right) \geq 0 \text {. }
$$

We recall that every bivariate copula is a restriction to the unit square $[0,1]^{2}$ of the cumulative distribution function of a vector $\left(U_{1}, U_{2}\right)$ of standard uniform random variables, i.e., for all $u_{1}, u_{2} \in[0,1], C\left(u_{1}, u_{2}\right)=$ $\operatorname{Pr}\left(U_{1} \leq u_{1}, U_{2} \leq u_{2}\right)$ and for $i=1,2, \operatorname{Pr}\left(U_{i} \leq u_{i}\right)=u_{i}$. Such random variables $U_{1}, U_{2}$ will be called representers of the copula $C$. Indeed, such a pair serves as a representative of the equivalence class of the pairs of random variables which are uniformly distributed on the unit interval $(0,1)$ and admit $C$ as their copula.

Due to Sklar's Theorem, the joint distribution function $F$ of any pair $(X, Y)$ of random variables defined on the probability space $(\Omega, \mathcal{M}, \mathbb{P})$ can be written as a composition of a copula $C$ and the univariate marginals $F_{1}$ and $F_{2}$, i.e. for all $(x, y) \in \mathbb{R}^{2}, F(x, y)=C\left(F_{1}(x), F_{2}(y)\right)$. Moreover, if $(X, Y)$ are continuous random variables, then the copula $C$ is uniquely determined.

We will denote the set of all two-dimensional copulas by $\mathcal{C}^{2}$. Note, that $\mathcal{C}^{2}$ is a bounded, compact, convex subset of the Banach space of the continuous functions on the unit square endowed with the supremum metric. In this paper we show that sometimes it is useful to consider the space of copulas with a more refined topology (compare [27]) and a more refined embedding into a Banach space. For more details about copula theory and some of its applications, we refer to $([5,8,14-17,20])$.

Note that the copulas are weakly differentiable. We recall that a function $\psi\left(x_{1}, x_{2}\right)$, defined on an open set $U$, is called a weak partial derivative of a function $\Psi\left(x_{1}, x_{2}\right)$ with respect to the $i$ th variable, written

$$
D_{i} \Psi=\psi
$$

provided

$$
\int_{U} \psi\left(x_{1}, x_{2}\right) h\left(x_{1}, x_{2}\right) d x_{1} d x_{2}=-\int_{U} \Psi\left(x_{1}, x_{2}\right) \frac{\partial h\left(x_{1}, x_{2}\right)}{\partial x_{i}} d x_{1} d x_{2}
$$

for all infinitely many times differentiable test functions with compact support $h, h \in C_{c}^{\infty}(U)$. To distinguish the classical partial derivatives from their weak generalizations, we will denote the first ones by $\frac{\partial}{\partial v_{i}}$ and the second ones by $D_{i}$.

Since copulas are Lipschitz functions, they are differentiable except possibly some set of Lebesgue measure 
0 . Therefore any measurable extension of the partial derivative is a weak derivative.

$$
D_{i} C\left(v_{1}, v_{2}\right) \stackrel{a e}{=} \frac{\partial C\left(v_{1}, v_{2}\right)}{\partial v_{i}} .
$$

The set $H^{1}\left(\mathbb{R}^{2}\right)$ consisting of weakly differentiable functions $h$ defined on the real plane, such that $h$ and its weak derivatives $D_{1} h$ and $D_{2} h$ are square integrable, endowed with a scalar product

$$
\left\langle h_{1}, h_{2}\right\rangle=\int_{\mathbb{R}^{2}}\left(h_{1}(x, y) h_{2}(x, y)+D_{1} h_{1}(x, y) D_{1} h_{2}(x, y)+D_{2} h_{1}(x, y) D_{2} h_{2}(x, y)\right) d x d y
$$

and a norm

$$
\|h\|_{H^{1}}=\sqrt{\|h\|_{L^{2}}^{2}+\left\|D_{1} h\right\|_{L^{2}}^{2}+\left\|D_{2} h\right\|_{L^{2}}^{2}},
$$

is a Hilbert space, an example of the Sobolev spaces. For more details the reader is referred for example to $[3,9,11]$.

In the following we associate with every copula a continuous linear functional on $H^{1}\left(\mathbb{R}^{2}\right)$ i.e. an element of the dual space $H^{1}\left(\mathbb{R}^{2}\right)^{\star}$

$$
C \hookrightarrow \int_{[0,1]^{2}} h(x, y) C(x, y) d x d y .
$$

For our purposes the best suited is the * weak topology on $H^{1}\left(\mathbb{R}^{2}\right)^{\star}$.

We say that a family of copulas $\left(C_{t}\right)_{t \geq 0}$ is differentiable at a point $t$ with respect to *weak topology on $H^{1}\left(\mathbb{R}^{2}\right)^{\star}$ if and only if for every test function $h \in H^{1}\left(\mathbb{R}^{2}\right)$ the limit

$$
\lim _{\delta \rightarrow 0} \int_{[0,1]^{2}} h\left(v_{1}, v_{2}\right) \frac{C_{t+\delta}\left(v_{1}, v_{2}\right)-C_{t}\left(v_{1}, v_{2}\right)}{\delta} d v_{1} d v_{2}
$$

exists.

Next when we are dealing not just with one one-parameter family but with a family of one-parameter families $\mathfrak{C}$, we require that it has a form of a flow, i.e. there exists a mapping

$$
\Lambda: \mathbb{R}^{+} \longrightarrow \operatorname{End}\left(\mathbb{R}^{+}, \mathrm{C}^{2}\right),
$$

such that:

1. $\Lambda_{0}$ is the identity mapping and the family of endomorphisms $\left(\Lambda_{t}\right)_{t \geq 0}$ with composition is a semigroup

$$
\Lambda_{t}\left(\Lambda_{s}(u, C)\right)=\Lambda_{t+s}(u, C) .
$$

2. Graphs of the families from $\mathfrak{C}$ are images of orbits of $\Lambda$

$$
t \hookrightarrow \Lambda_{t}(u, C) .
$$

Since for $\delta>0$

$$
\frac{1}{\delta}\left(\Lambda_{t+\delta}(s, C)-\Lambda_{t}(s, C)\right)=\left(\frac{1}{\delta}\left(\Lambda_{\delta}-\Lambda_{0}\right)\right)\left(\Lambda_{t}(s, C)\right),
$$

the tangent vectors to graphs of the families from $\mathfrak{C}$ are described in terms of the infinitesimal generator of $\Lambda$.

\section{Preliminaries}

We consider a probability space $(\Omega, \mathcal{M}, \mathbb{P})$, with filtration $\mathcal{F}=\left\{\mathcal{F}_{t}\right\}_{t \geq 0}$ and a pair of randomly correlated Wiener processes $W^{1}=\left(W_{t}^{1}\right)_{t \geq 0}$ and $W^{2}=\left(W_{t}^{2}\right)_{t \geq 0}$.

We assume:

A1. $W^{1}$ and $W^{2}$ are adapted to the filtration $\mathcal{F}$ and the increments $W_{t+h}^{i}-W_{t}^{i}, i=1,2$, are all independent of 
every event in $\mathcal{F}_{t}$ for $t \geq 0$ and $h>0$.

Let $\mathcal{F}$ adapted path-continuous processes $\left(X^{1}, X^{2}\right)=\left(X_{t}^{1}, X_{t}^{2}\right)_{t \geq 0}$, be solutions of the following system of stochastic differential equations:

$$
\begin{aligned}
d X_{t}^{1} & =\mu_{1}\left(t, X_{t}^{1}\right) d t+\sigma_{1}\left(t, X_{t}^{1}\right) d W_{t}^{1}, \\
d X_{t}^{2} & =\mu_{2}\left(t, X_{t}^{2}\right) d t+\sigma_{2}\left(t, X_{t}^{2}\right) d W_{t}^{2} .
\end{aligned}
$$

We understand the above as integral equations:

$$
X_{t_{2}}^{i}=X_{t_{1}}^{i}+\int_{t_{1}}^{t_{2}} \mu_{i}\left(s, X_{s}^{i}\right) d s+\int_{t_{1}}^{t_{2}} \sigma_{i}\left(s, X_{s}^{i}\right) d W_{s}^{i}, \quad t_{2}>t_{1} \geq 0, \quad i=1,2,
$$

where

A2. $\mu_{i}(t, x)$ and $\sigma_{i}(t, x)$ are continuous functions defined on $[0,+\infty) \times(-\infty,+\infty)$, continuously differentiable with respect to $x$ with bounded derivatives

$$
\forall T>0 \quad \exists L \in \mathbb{R}^{+} \quad \forall x \in \mathbb{R} \quad \forall t \in[0, T] \quad\left|\frac{\partial \mu_{i}(t, x)}{\partial x}\right|+\left|\frac{\partial \sigma_{i}(t, x)}{\partial x}\right|<L, \quad i=1,2 .
$$

For more details the reader is referred to the extensive literature on SDE, for example [1, 4, 10, 18, 21, 24]. Furthermore we assume:

A3. The quadratic covariation of $X^{1}$ and $X^{2}$ can be described by a deterministic function of $X^{1}$ and $X^{2}$

$$
d\left\langle X^{1}, X^{2}\right\rangle_{t}=\sigma_{1}\left(t, X_{t}^{1}\right) \sigma_{2}\left(t, X_{t}^{2}\right) A\left(t, X_{t}^{1}, X_{t}^{2}\right) d t=\sigma_{1}\left(t, X_{t}^{1}\right) \sigma_{2}\left(t, X_{t}^{2}\right) d\left\langle W^{1}, W^{2}\right\rangle_{t},
$$

where $|A|$ is a bounded by 1 and the product $\sigma_{2}\left(t, x_{2}\right) A\left(t, x_{1}, x_{2}\right)$ is differentiable with respect to $x_{2}$ with bounded derivative

$$
\forall T>0 \quad \exists L_{A} \in \mathbb{R}^{+} \quad \forall\left(x_{1}, x_{2}\right) \in \mathbb{R}^{2} \quad \forall t \in[0, T] \quad\left|\frac{\partial \sigma_{2}\left(t, x_{2}\right) A\left(t, x_{1}, x_{2}\right)}{\partial x_{2}}\right|<L_{A} .
$$

A4. The univariate distribution functions $F_{i}(t, x)=P\left(X_{t}^{i} \leq x\right)$ are differentiable with respect to $t$, for $t>0$, right-side differentiable at $t=0$ and twice differentiable with respect to $x$, with positive first derivatives with respect to $x$. The derivatives are continuous with respect to both variables and "regular" at $\pm \infty$, i.e. there exists $\varepsilon>0$ such that for $i=1,2$, almost uniformly with respect to $t$ :

$$
\begin{aligned}
\lim _{x \rightarrow \pm \infty} \frac{\partial F_{i}(t, x)}{\partial t} & =0, \\
\lim _{x \rightarrow \pm \infty} x \frac{\partial F_{i}(t, x)}{\partial x} & =0, \\
\lim _{x \rightarrow \pm \infty}|x|^{2+\varepsilon} \frac{\partial^{2} F_{i}(t, x)}{\partial x^{2}} & =0 .
\end{aligned}
$$

Let $C_{t}$ be a corresponding copula process

$$
P\left(X_{t}^{1} \leq x_{1}, X_{t}^{2} \leq x_{2}\right)=C_{t}\left(F_{1}\left(t, x_{1}\right), F_{2}\left(t, x_{2}\right)\right) .
$$

We set for $i=1,2$

$$
U_{i, t}=F_{i}\left(t, X_{t}^{i}\right)
$$

Obviously $U_{1, t}$ and $U_{2, t}$ are uniformly distributed and are the representers of the copula $C_{t}$. The processes of representers $U_{1}$ and $U_{2}$ are also called the copula uniform marginal processes.

Since the assumption A4. allows us to apply the Ito's Lemma, there exist functions $v^{i}(t, u)$ such that

$$
d U_{i, t}=v_{i, 1}\left(t, U_{i, t}\right) d t+v_{i, 2}\left(t, U_{i, t}\right) d W_{t}^{i} .
$$


Indeed from Ito's Lemma we get for $i=1,2$

$$
\begin{aligned}
& v_{i, 1}\left(t, F_{i}(t, x)\right)=\frac{\partial F_{i}(t, x)}{\partial t}+\frac{\partial F_{i}(t, x)}{\partial x} \mu_{i}(t, x)+\frac{1}{2} \frac{\partial^{2} F_{i}(t, x)}{\partial x^{2}} \sigma_{i}^{2}(t, x), \\
& v_{i, 2}\left(t, F_{i}(t, x)\right)=\frac{\partial F_{i}(t, x)}{\partial x} \sigma_{i}(t, x) .
\end{aligned}
$$

Since by $\mathbf{A} 4$ the univariate distribution functions $F_{i}$ are strictly increasing, the above defines $v$ 's inside the unit interval. We extend $v$ 's by 0 . For $u \leq 0$ and $u \geq 1$ we set

$$
v_{i, 1}(t, u)=v_{i, 2}(t, u)=0
$$

The last part of $\mathbf{A} 4$ and $\mathbf{A} 2$ imply that constructed in such a way $v_{i, j}$ are continuous functions on $[0,+\infty) \times \mathbb{R}$. Hence they are bounded on compact subsets of $t$ i.e. on $[0, T] \times \mathbb{R}$. Moreover, since $F_{i}$ have differentiable inverses, the volatilities $v_{i, 2}$ are differentiable with respect to $x$ on the whole real line, except perhaps at the two points 0 and 1 .

Note that

$$
d\left\langle U_{1}, U_{2}\right\rangle_{t}=v_{1,2}\left(t, U_{1, t}\right) v_{2,2}\left(t, U_{2, t}\right) A_{C}\left(t, U_{1, t}, U_{2, t}\right) d t
$$

where

$$
A_{C}\left(t, F_{1}\left(t, x_{1}\right), F_{2}\left(t, x_{2}\right)\right)=A\left(t, x_{1}, x_{2}\right)
$$

Indeed

$$
\begin{aligned}
d\left\langle U_{1}, U_{2}\right\rangle_{t} & =v_{1,2}\left(t, U_{1, t}\right) v_{2,2}\left(t, U_{2, t}\right) d\left\langle W^{1}, W^{2}\right\rangle_{t} \\
& =\frac{\partial F_{1}\left(t, X_{t}^{1}\right)}{\partial x} \sigma_{1}\left(t, X_{t}^{1}\right) \frac{\partial F_{2}\left(t, X_{t}^{2}\right)}{\partial x} \sigma_{i}\left(t, X_{t}^{2}\right) d\left\langle W^{1}, W^{2}\right\rangle_{t} \\
& =\frac{\partial F_{1}\left(t, X_{t}^{1}\right)}{\partial x} \sigma_{1}\left(t, X_{t}^{1}\right) \frac{\partial F_{2}\left(t, X_{t}^{2}\right)}{\partial x} \sigma_{i}\left(t, X_{t}^{2}\right) A\left(t, X_{t}^{1}, X_{t}^{2}\right) d t \\
& =v_{1,2}\left(t, U_{1, t}\right) v_{2,2}\left(t, U_{2, t}\right) A_{C}\left(t, U_{1, t}, U_{2, t}\right) d t
\end{aligned}
$$

Drift and volatility of SDE for representers are closely related.

Proposition 3.1. If the processes of representers $\left(U_{j, t}\right)_{t \geq 0}, j=1,2$, fulfill equations (12) then for $s \geq 0$

$$
\int_{0}^{1} v_{j, 1}(s, \xi) d \xi=0
$$

and for $u \in(0,1)$

$$
v_{j, 2}(s, u) \frac{\partial v_{j, 2}(s, u)}{\partial u}=v_{j, 1}(s, u)
$$

The proof of the proposition is provided in section 6.1.

In the example below we show that the volatilities $v_{i, 2}(t, x)$ need not to be differentiable at $x=0,1$.

Example 3.1. The Gaussian copula.

Let $\left(X^{1}, X^{2}\right)$ be a shifted by 1 two-dimensional Wiener process. Since for every $t \geq 0$ the pair $\left(X_{t}^{1}, X_{t}^{2}\right)$ has a bivariate normal distribution and the correlation is constant, the corresponding copula is a Gaussian one (see [8] section 6.7 for more details). Such a process fulfills assumptions A1 - A4 with the following parameters and initial values:

$$
\mu_{i}=0, \quad \sigma_{i}=1, \quad A(t, x, y)=\rho, \quad\left(X_{0}^{1}, X_{0}^{2}\right) \sim N(0, \Sigma), \quad \Sigma=\left(\begin{array}{cc}
1 & \rho \\
\rho & 1
\end{array}\right) .
$$

For $u \in(0,1)$ we have

$$
v_{i, 1}(t, u)=-\varphi\left(\Phi^{-1}(u)\right) \frac{\Phi^{-1}(u)}{1+t}
$$




$$
\begin{aligned}
v_{i, 2}(t, u) & =\varphi\left(\Phi^{-1}(u)\right) \frac{1}{\sqrt{1+t}}, \\
A_{C}\left(t, u_{1}, u_{2}\right) & =\rho,
\end{aligned}
$$

where $\Phi$ and $\varphi$ are the distribution function and the density of the standard normal probability law $N(0,1)$. Note that for $u \in(0,1)$

$$
\frac{d}{d u} \varphi\left(\Phi^{-1}(u)\right)=-\Phi^{-1}(u)
$$

Furthermore both functions $\Phi^{-1}(u)$ and $\varphi\left(\Phi^{-1}(u)\right)$ are regularly varying at $0^{+}$and $1^{-}$. The first one is slowly varying, while the second has index of variation equal 1. For more details the reader is referred to section 6.2. Note that for $i, j=1,2$

$$
\lim _{u \rightarrow 0^{+}} v_{i, j}(t, u)=\lim _{u \rightarrow 1^{-}} v_{i, j}(t, u)=0,
$$

and, although $v_{i, j}(t, u)$ are not differentiable at $O$ and 1 , they are regularly varying with index of variation 1.

\section{The Copula processes}

We have defined a one-parameter family of copulas $\left(C_{t}\right)_{t \geq 0}$, i.e. a curve:

$$
\gamma:[0,+\infty) \longrightarrow \mathrm{e}^{2}, \quad \gamma(t)=C_{t} .
$$

Problem:

Choose an embedding of $\mathrm{C}^{2}$ into a topological linear space such that the embedded family is not only continuous but also differentiable with respect to the parameter $t$.

We provide the solution of this problem by embedding the space of copulas into the space of continuous linear functionals on the Hilbert space of Sobolev functions on the real plane $H^{1}\left(\mathbb{R}^{2}\right)$.

The embedding

$$
j: \mathcal{C}^{2} \longrightarrow H^{1}\left(\mathbb{R}^{2}\right)^{*}
$$

is constructed in the following way

$$
j(C)(h)=\int_{[0,1]^{2}} h(x, y) C(x, y) d x d y .
$$

Note that since copulas are bounded by 1 , we have for every $h \in H^{1}\left(\mathbb{R}^{2}\right)$

$$
\begin{aligned}
|j(C)(h)| & \leq \int_{[0,1]^{2}}|h(x, y)| C(x, y) d x d y \\
& \leq\left(\int_{[0,1]^{2}} h(x, y)^{2} d x d y\right)^{1 / 2}\left(\int_{[0,1]^{2}} C(x, y)^{2} d x d y\right)^{1 / 2} \\
& \leq\left(\int_{[0,1]^{2}} h(x, y)^{2} d x d y\right)^{1 / 2} \leq\|h\|_{L^{2}} \leq\|h\|_{H^{1}} .
\end{aligned}
$$

Hence $j(C)$ is indeed a continuous linear functional. Furthermore the mapping

$$
j\left(C_{t}\right):[0, \infty) \longrightarrow H^{1}\left(\mathbb{R}^{2}\right)^{\star}
$$

is differentiable with respect to the ${ }^{\star}$ weak topology on $H^{1}\left(\mathbb{R}^{2}\right)^{\star}$ (for $t=0$ we take the right-side derivative). 
Theorem 4.1. For any $h \in H^{1}\left(\mathbb{R}^{2}\right)$ and $t \geq 0$

$$
\frac{d}{d t} \int_{[0,1]^{2}} h\left(v_{1}, v_{2}\right) C_{t}\left(v_{1}, v_{2}\right) d v_{1} d v_{2}=-B_{t}\left(h, C_{t}\right),
$$

where for fixed $t \geq 0$ and fixed copula $C, B_{t}(\cdot, C)$ is a continuous linear functional on $H^{1}\left(\mathbb{R}^{2}\right)$, given by a formula

$$
\begin{aligned}
B_{t}(h, C) & =\frac{1}{2} \int_{[0,1]^{2}} D_{1}\left(h\left(v_{1}, v_{2}\right) v_{1,2}\left(t, v_{1}\right)^{2}\right) D_{1} C\left(v_{1}, v_{2}\right) d v_{1} d v_{2} \\
& +\frac{1}{2} \int_{[0,1]^{2}} D_{2}\left(h\left(v_{1}, v_{2}\right) v_{2,2}\left(t, v_{2}\right)^{2}\right) D_{2} C\left(v_{1}, v_{2}\right) d v_{1} d v_{2} \\
& +\int_{[0,1]^{2}} D_{2}\left(h\left(v_{1}, v_{2}\right) v_{1,2}\left(t, v_{1}\right) v_{2,2}\left(t, v_{2}\right) A_{c}\left(t, v_{1}, v_{2}\right)\right) D_{1} C\left(v_{1}, v_{2}\right) d v_{1} d v_{2}
\end{aligned}
$$

The proof is provided in section 6.3.

Since $B_{t}$ is uniformly bounded on bounded sets of $t, j\left(C_{t}\right)$ is locally Lipschitz in respect to the strong topology on $H^{1}\left(\mathbb{R}^{2}\right)^{\star}$.

Theorem 4.2. For any $T>0$ and $s, t \in[0, T]$ there exists a constant $L_{T}$ such that for all $h \in H^{1}$

$$
\left|\int_{[0,1]^{2}} h\left(v_{1}, v_{2}\right) C_{t}\left(v_{1}, v_{2}\right) d v_{1} d v_{2}-\int_{[0,1]^{2}} h\left(v_{1}, v_{2}\right) C_{s}\left(v_{1}, v_{2}\right) d v_{1} d v_{2}\right| \leq L_{T}|| h||_{H^{1}}|t-s| .
$$

The proof is provided in section 6.3.

When the copula $C$ is twice differentiable, the above can be restated in terms of partial differential equations. Applying integration by parts with respect to respectively the first and the second variable, we get:

Corollary 4.1. For $C$ twice differentiable on $(0,1)^{2}$, any test function $h$ with support contained in $(0,1)^{2}$ and $t \geq 0$

$$
\begin{aligned}
B_{t}(h, C) & =-\int_{[0,1]^{2}} h\left(v_{1}, v_{2}\right)\left(\frac{1}{2} \sum_{j=1}^{2} v_{j, 2}^{2}\left(t, v_{j}\right) \frac{\partial^{2} C\left(v_{1}, v_{2}\right)}{\partial v_{j}^{2}}\right. \\
& \left.+v_{1,2}\left(t, v_{1}\right) v_{2,2}\left(t, v_{2}\right) A_{C}\left(t, v_{1}, v_{2}\right) \frac{\partial^{2} C\left(v_{1}, v_{2}\right)}{\partial v_{1} \partial v_{2}}\right) d v_{1} d v_{2}
\end{aligned}
$$

Therefore, using the terminology of the theory of partial differential equations, we may say that the family $C_{t}$, defined by (10), is a weak solution ([9]) of a parabolic differential equation

$$
\begin{aligned}
\frac{\partial C\left(t, v_{1}, v_{2}\right)}{\partial t} & =\frac{1}{2} \sum_{j=1}^{2} v_{j, 2}^{2}\left(t, v_{j}\right) \frac{\partial^{2} C\left(t, v_{1}, v_{2}\right)}{\partial v_{j}^{2}} \\
& +v_{1,2}\left(t, v_{1}\right) v_{2,2}\left(t, v_{2}\right) A_{C}\left(t, v_{1}, v_{2}\right) \frac{\partial^{2} C\left(t, v_{1}, v_{2}\right)}{\partial v_{1} \partial v_{2}}
\end{aligned}
$$

Note that equation (30) was already studied in literature, but under more demanding assumptions. In [6] it was proved for copulas $C$ with twice differentiable density, three times differentiable marginals $F_{i}$ and the interdependence given by a quadratic correlation function $A\left(t, x_{1}, x_{2}\right)$, introduced in $\mathbf{A} \mathbf{3}$, being a product

$$
A\left(t, x_{1}, x_{2}\right)=A_{1}\left(t, x_{1}\right) A_{2}\left(t, x_{2}\right) \text {. }
$$


In [12] a stationary version of (30) was derived for twice differentiable copulas and Brownian margins.

To illustrate the possible applications of Theorem 4.1 we provide the following example concerning comonotonic random processes. We recall that random variables are comonotonic when they admit a singular copula $M$ as their copula

$$
M\left(v_{1}, v_{2}\right)=\min \left(v_{1}, v_{2}\right) .
$$

For more details the reader is referred to $[8]$ Theorem 2.5.7.

Proposition 4.1. Let the stochastic processes $X^{1}$ and $X^{2}$ be as in section 3 and $T>0$ be a fixed horizon. If for all $t \in[0, T]$ the random variables $X_{t}^{1}$ and $X_{t}^{2}$ are comonotonic then for all $t \in[0, T]$ and $v \in[0,1]$

$$
\left|v_{1,2}(t, v)\right|=\left|v_{2,2}(t, v)\right| \quad \text { and } \quad v_{1,2}(t, v)=v_{2,2}(t, v) A_{C}(t, v, v) \text {. }
$$

The proof is based on the study of the dynamic of $\rho\left(C_{t}\right)$, where $\rho$ is the Spearman rank correlation coefficient. It is provided in subsection 6.4.

\section{A semigroup approach to copula processes}

The theory of semigroups of operators plays an important role in two domains which were mentioned in the previous sections, diffusion processes described by stochastic differential equations and parabolic partial differential equations (see for example [9, 21]). We show that it might be applied to the study of copula processes as well. To do it, we have to add two more assumptions which will ensure the existence and uniqueness, necessary to construct a semigroup of transformations.

A5. The functions $\sigma_{2} A$ and $\sigma_{2} B$, where $B=\sqrt{1-A^{2}}$, are Lipschitz.

$$
\begin{aligned}
& \forall T>0 \quad \exists L_{A B} \quad \forall\left(x_{1}, x_{2}\right),\left(y_{1}, y_{2}\right) \in \mathbb{R}^{2} \quad \forall t \in[0, T] \\
& \left|\sigma_{2}\left(t, x_{2}\right) A\left(t, x_{1}, x_{2}\right)-\sigma_{2}\left(t, y_{2}\right) A\left(t, y_{1}, y_{2}\right)\right|+\left|\sigma_{2}\left(t, x_{2}\right) B\left(t, x_{1}, x_{2}\right)-\sigma_{2}\left(t, y_{2}\right) B\left(t, y_{1}, y_{2}\right)\right| \\
& \leq L_{A B}\left(\left|x_{1}-y_{1}\right|+\left|x_{2}-y_{2}\right|\right) .
\end{aligned}
$$

A6. The functions $\sigma_{1}$ and $\sigma_{2}$ do not vanish and the supremum of $|A|$ is less than 1 .

$$
\begin{aligned}
& \forall T>0 \quad \forall x_{1}, x_{2} \in \mathbb{R} \quad \forall t \in[0, T] \quad \sigma_{1}\left(t, x_{1}\right) \sigma_{2}\left(t, x_{2}\right) \neq 0, \\
& \forall T>0 \quad \exists A_{M} \quad \forall\left(x_{1}, x_{2}\right) \in \mathbb{R}^{2} \quad \forall t \in[0, T] \quad\left|A\left(t, x_{1}, x_{2}\right)\right| \leq A_{M}<1 .
\end{aligned}
$$

Theorem 5.1. Let a pair of stochastic processes $X^{1}$ and $X^{2}$ fulfill A1. - A6. with parameters $\mu_{i}, \sigma_{i}, F_{i}, i=1,2$, and $A$. Then for any copula $C$ and $s \geq 0$ there exists a filtered probability space, a pair of Wiener processes and two stochastic processes $X^{1, s, C}$, and $X^{2, s, C}$ fulfilling A1. - A4. with shifted parameters

$$
\begin{aligned}
& \tilde{\mu}_{i}(t, x)=\mu(t+s, x), \quad \tilde{\sigma}(t, x)=(t+s, x), \\
& \tilde{F}_{i}(t, x)=F_{i}(t+s, x), \quad \tilde{A}\left(t, x_{1}, x_{2}\right)=A\left(t+s, x_{1}, x_{2}\right),
\end{aligned}
$$

such that $C$ is the copula of $\left(X_{0}^{1, s, C}, X_{0}^{2, s, C}\right)$.

The pair of stochastic processes $\left(X^{1, s, C}, X^{2, s, C}\right)$ is law-unique.

The proof is provided in section 6.5.

We denote the copula corresponding to the pair $\left(X_{t}^{1, s, C}, X_{t}^{2, s, C}\right)$ by $C_{t}^{s, C}$.

Since the pair $\left(X^{1, s, C}, X^{2, s, C}\right)$ is law-unique, the copula process $\left(C_{t}^{s, C}\right)_{t \geq 0}$ is well defined. It does not depend on the choice of the probability space, Wiener processes and the pair $\left(X^{1, s, C}, X^{2, s, C}\right)$, as long as the assumptions of the theorem are fulfilled. 
Moreover, since the restricted process $\left(X_{t_{1}+t}^{1, s, C}, X_{t_{1}+t}^{2, s, C}\right)_{t \geq 0}, t_{1}>0$, coincides with the process with parameters shifted by $t_{1}+s$ and initial copula equal copula of the pair $\left(X_{t_{1}}^{1, s, C}, X_{t_{1}}^{2, s, C}\right)$ (i.e. $\left.C_{t_{1}}^{s, C}\right)$

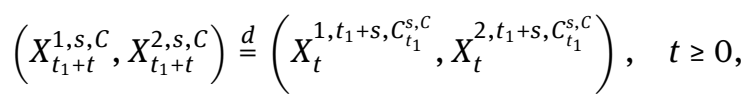

we get a chain rule for copulas

$$
C_{t}^{t_{1}+s, C_{t_{1}}^{s, C}}=C_{t_{1}+t}^{s, C}
$$

The restricted copula process $\left(C_{t_{1}+t}^{s, C}\right)_{t \geq 0}, t_{1}>0$, coincides with the copula process with parameters shifted by $t_{1}+s$ and initial copula equal copula $C_{t_{1}}^{s, C}$.

Now we are in a position to define a semigroup of transformations $\Lambda$. To make our system "autonomous" we add one more dependent variable. Instead of a process $\left(C_{t}\right)_{t \geq 0}$, we consider $\left(t, C_{t}\right)_{t \geq 0}$. We set

$$
\Lambda: \mathbb{R}^{+} \longrightarrow \operatorname{End}\left(\mathbb{R}^{+} \times \mathcal{C}^{2}\right) ; \quad \Lambda_{t}(s, C)=\left(t+s, C_{t}^{s, C}\right) .
$$

Here End denotes the set of endomorphisms. The above chain rule for copulas implies that $\Lambda$ is a semigroup. Indeed:

$$
\begin{gathered}
\Lambda_{t_{2}} \circ \Lambda_{t_{1}}(s, C)=\Lambda_{t_{2}}\left(t_{1}+s, C_{t_{1}}^{s, C}\right) \\
=\left(t_{2}+t_{1}+s, C^{t_{1}+s, C_{t_{1}}^{s, C}}\right)=\left(t_{2}+t_{1}+s, C_{t_{2}+t_{1}}^{s, C}\right)=\Lambda_{t_{2}+t_{1}}(s, C) .
\end{gathered}
$$

Note that the graph of the copula process $C_{t}$ corresponding to the pair $\left(X^{1}, X^{2}\right)$ is an orbit of $\Lambda$. Indeed

$$
\Lambda_{t}\left(0, C_{0}\right)=\left(t, C_{t}\right)
$$

We show that that local properties of $\Lambda$ can be described in terms of the bilinear form $B$ introduced by (27). In order to be able to apply the concept of differentiability, we imbed our state space into a Banach space

$$
(i d, j): \mathbb{R}^{+} \times \mathrm{C}^{2} \longrightarrow \mathbb{R} \times H^{1}\left(\mathbb{R}^{2}\right)^{\star},
$$

where $j$ is given by formula (24). We select the * weak topology on $\left(H^{1}\right)^{\star}$. Following the notation used for semigroups of linear operators we introduce an infinitesimal generator $L$

$$
\begin{aligned}
L: & D(L) \longrightarrow \mathbb{R} \times H^{1}\left(\mathbb{R}^{2}\right)^{\star}, \quad D(L) \subset \mathbb{R}^{+} \times \mathcal{C}^{2}, \\
& \lim _{t \rightarrow 0^{+}} \frac{(i d, j) \circ \Lambda_{t}(s, C)-(s, j(C))}{t}-L(s, C)=0 .
\end{aligned}
$$

It shows that the choice of the embedding $j$ and the ${ }^{\star}$ weak topology ensures that $(i d, j) \circ \Lambda_{t}(s, C)$ is differentiable for all $(s, C)$, i.e. $D(L)=\mathbb{R}^{+} \times \mathcal{C}^{2}$.

Theorem 5.2. With respect to the topology induced by imbedding $j: \mathrm{e}^{2} \rightarrow\left(H^{1}\left(\mathbb{R}^{2}\right)\right)^{\star}, \Lambda$ is a strongly continuous semigroup of transformations. Moreover the generator $L$ is a mapping given by

$$
L: \mathbb{R}^{+} \times \mathrm{C}^{2} \longrightarrow \mathbb{R} \times H^{1}\left(\mathbb{R}^{2}\right)^{\star}, \quad L(s, C)=\left(1,-B_{s, C}\right),
$$

where for a test function $h \in H^{1}\left(\mathbb{R}^{2}\right)$

$$
B_{s, C}(h)=B_{s}(h, C) \text {. }
$$

The proof is provided in section 6.5 .

Since the graph of the copula process $C_{t}$ corresponding to the pair $\left(X^{1}, X^{2}\right)$ is an orbit of $\Lambda$

$$
\Lambda_{t}\left(0, C_{0}\right)=\left(t, C_{t}\right) \text {, }
$$

the tangent vector to the curve $t \rightarrow j\left(C_{t}\right)$ and the generator of the semigroup coincide.

$$
L\left(s, C_{s}\right)=\left(1, \frac{d}{d t} j\left(C_{t}\right)_{\mid t=s}\right) .
$$




\section{Proofs and auxiliary results}

\subsection{Proof of Proposition 3.1}

Since both drifts $v_{j, 2}$ are bounded,

$$
E\left(U_{j, t}\right)-E\left(U_{j, 0}\right)=\int_{0}^{t} E\left(v_{j, 1}\left(s, U_{s}^{j}\right)\right) d s=\int_{0}^{t}\left(\int_{0}^{1} v_{j, 1}(s, u) d u\right) d s .
$$

Since the expected values of all $U_{j, t}$ are the same, we get for $s>0$

$$
\int_{0}^{1} v_{j, 1}(s, u) d u=0 .
$$

Next, we calculate the characteristic function of the random variable $U_{j, t}, t \geq 0$.

$$
d \exp \left(i z U_{j, t}\right)=\left(i z v_{j, 1} \exp \left(i z U_{j, t}\right)-\frac{1}{2} z^{2} v_{j, 2}^{2} \exp \left(i z U_{j, t}\right)\right) d t+\exp \left(i z U_{j, t}\right) v_{j, 2} d W_{t}^{j} .
$$

Since $v_{j, 2}$ are bounded,

$$
E\left(\exp \left(i z U_{j, t}\right)\right)=E\left(\exp \left(i z U_{j, 1}\right)\right)+\int_{0}^{t} E\left(i z v_{j, 1} \exp \left(i z U_{s}^{j}\right)-\frac{1}{2} z^{2} v_{j, 2}^{2} \exp \left(i z U_{s}^{j}\right)\right) d s
$$

Furthermore, since all $U_{s}^{j}$ are uniformly distributed on [0, 1], the characteristic function does not depend on $s$ and we get for all $z \in \mathbb{R}$

$$
\begin{aligned}
0 & =E\left(i z v_{j, 1} \exp \left(i z U_{s}^{j}\right)-\frac{1}{2} z^{2} v_{j, 2}^{2} \exp \left(i z U_{s}^{j}\right)\right) \\
& =i z \int_{0}^{1} v_{j, 1}(s, u) \exp (i z u) d u-\frac{1}{2} z^{2} \int_{0}^{1} v_{j, 2}(s, u)^{2} \exp (i z u) d u .
\end{aligned}
$$

We divide (40) by $z^{2}$ and and apply integration by parts to the first integral. We get for all $z \in \mathbb{R}$ :

$$
\int_{0}^{1}\left(\int_{0}^{u} v_{j, 1}(s, \xi) d \xi\right) \exp (i z u) d u-\frac{1}{2} \int_{0}^{1} v_{j, 2}(s, u)^{2} \exp (i z u) d u=0 .
$$

Hence for all $u \in[0,1]$ and $s \geq 0$

$$
2 \int_{0}^{u} v_{j, 1}(s, \xi) d \xi=v_{j, 2}(s, u)^{2}
$$

Since $v_{j, 1}$ are continuous and $v_{j, 2}$ are differentiable for $u \in(0,1)$, the above implies

$$
v_{j, 2}(s, u) \frac{\partial v_{j, 2}(s, u)}{\partial u}=v_{j, 1}(s, u)
$$

for $s \geq 0$ and $u \in(0,1)$. 


\subsection{A note on regular variation}

We recall that a function $U:(0,+\infty) \rightarrow(0,+\infty)$ is regularly varying at $+\infty$ with index $\rho \in[-\infty,+\infty]$ if for every $x>0$ the limit

$$
\lim _{t \rightarrow+\infty} \frac{U(t x)}{U(t)}
$$

exists and equals $x^{\rho}$, where

$$
x^{+\infty}=\left\{\begin{array}{ccc}
0 & \text { if } & x<1 \\
1 & \text { if } & x=1 \\
+\infty & \text { if } & x>1
\end{array}\right.
$$

and

$$
x^{-\infty}=\left\{\begin{array}{ccc}
+\infty & \text { if } & x<1 \\
1 & \text { if } & x=1 \\
0 & \text { if } & x>1
\end{array}\right.
$$

(compare [22] Definition 2.1 and section 2.4). The variation with index 0 is also called the slow variation, while variation with index $+\infty$ - the rapid variation.

The above can be extended for functions $F:(0,1) \rightarrow(-\infty,+\infty)$. We say that $F$ is regularly varying at $0^{+}$ or respectively $1^{-}$with index $\rho \in[-\infty,+\infty]$ if for every $x>0$

$$
\lim _{t \rightarrow 0^{+}} \frac{F(t x)}{F(t)}=x^{\rho},
$$

or respectively

$$
\lim _{t \rightarrow 0^{+}} \frac{F(1-t x)}{F(1-t)}=x^{\rho} .
$$

Let $\Phi, \Phi^{-1}$ and $\varphi$ denote the distribution function, the quantile function and the density of the standard normal distribution $N(0,1)$.

Lemma 6.1. The quantile function $\Phi^{-1}:(0,1) \rightarrow(-\infty,+\infty)$ is slowly varying at $0^{+}$and $1^{-}$. For $x>0$

$$
\lim _{t \rightarrow 0^{+}} \frac{\Phi^{-1}(t x)}{\Phi^{-1}(t)}=\lim _{t \rightarrow 0^{+}} \frac{\Phi^{-1}(1-t x)}{\Phi^{-1}(1-t)}=1 .
$$

Proof.

First we show that the auxiliary function $U$,

$$
U(x)=\frac{1}{1-\Phi(x)},
$$

is rapidly varying at $+\infty$. Indeed applying the de l'Hospital rule we get

$$
\lim _{t \rightarrow+\infty} \frac{U(t x)}{U(t)} \stackrel{H}{=} \lim _{t \rightarrow+\infty} \frac{\varphi(t)}{x \varphi(t x)}=\lim _{t \rightarrow+\infty} \frac{1}{x} \exp \left(\frac{1}{2} t^{2}\left(x^{2}-1\right)\right)=\left\{\begin{array}{cll}
0 & \text { if } & x<1 \\
1 & \text { if } & x=1 \\
+\infty & \text { if } & x>1
\end{array}\right.
$$

Hence the inverse function $U^{-1}$ is slowly varying at $+\infty$ (see [22] Proposition 2.6.v). Since for $y \in(0,1)$

$$
-\Phi^{-1}(y)=\Phi^{-1}(1-y)=U^{-1}\left(\frac{1}{y}\right),
$$

$\Phi^{-1}$ is slowly varying at both ends of the unit interval. 
Lemma 6.2. The composition of the density $\varphi$ and the quantile function $\Phi^{-1}:(0,1) \rightarrow(-\infty,+\infty)$ is regularly varying at $0^{+}$and $1^{-}$with index 1 . For $x>0$

$$
\lim _{t \rightarrow 0^{+}} \frac{\varphi\left(\Phi^{-1}(t x)\right)}{\varphi\left(\Phi^{-1}(t)\right)}=\lim _{t \rightarrow 0^{+}} \frac{\varphi\left(\Phi^{-1}(1-t x)\right)}{\varphi\left(\Phi^{-1}(1-t)\right)}=x .
$$

Proof.

We apply the de l'Hospital rule and the previous lemma. Since

$$
\frac{d}{d y} \varphi\left(\Phi^{-1}(y)\right)=-\Phi^{-1}(y)
$$

we have

$$
\lim _{t \rightarrow 0^{+}} \frac{\varphi\left(\Phi^{-1}(t x)\right)}{\varphi\left(\Phi^{-1}(t)\right)} \stackrel{H}{=} \lim _{t \rightarrow 0^{+}} \frac{x \Phi^{-1}(t x)}{\Phi^{-1}(t)}=x .
$$

This finishes the proof because $\varphi$ is an even function and

$$
\varphi\left(\Phi^{-1}(1-y)\right)=\varphi\left(\Phi^{-1}(y)\right)
$$

\subsection{Proof of Theorems 4.1 and 4.2}

We start with showing that assumption $\mathbf{A} 3$ implies that, although the derivatives of $v_{2,2}$ might be unbounded, for fixed $v_{1}$ and $s>1$ the function

$$
G\left(v_{2}\right)=\frac{\partial}{\partial v_{2}}\left(v_{2,2}\left(s, v_{2}\right) A_{C}\left(s, v_{1}, v_{2}\right)\right)
$$

has finite $L^{1}$ norm.

Lemma 6.3. For any fixed $T>0$ there exists a constant $M_{T}$ such that for all $s \in[0, T]$ and $v_{1} \in(0,1)$

$$
\|G\|_{L^{1}}=\int_{0}^{1}\left|\frac{\partial}{\partial v_{2}}\left(v_{2,2}\left(s, v_{2}\right) A_{C}\left(s, v_{1}, v_{2}\right)\right)\right| d v_{2} \leq M_{T}<\infty .
$$

Proof.

We denote by $m$ the supremum of the value of $\left|\sigma_{2}(s, x) A\left(s, x_{1}, x\right)\right|$ at $x=0$. From the Mean Value Theorem we get an estimate for all $x$

$$
\left|\sigma_{2}(s, x) A\left(s, x_{1}, x\right)\right| \leq m+L_{A}|x| .
$$

Furthermore the assumption A4 implies that there exists a constant $M$ such that for all $x \in \mathbb{R}$ and $s \in[0, T]$

$$
\left|\frac{\partial^{2}}{\partial x^{2}} F_{2}(s, x)\right| \leq \frac{M}{1+|x|^{2+\varepsilon}} .
$$

Applying the above we get:

$$
\begin{aligned}
\|G\|_{L^{1}} & =\int_{0}^{1}\left|G\left(v_{2}\right)\right| d v_{2} \\
& =\int_{-\infty}^{+\infty}\left|G\left(F_{2}(s, x)\right)\right| \frac{\partial}{\partial x} F_{2}(s, x) d x
\end{aligned}
$$




$$
\begin{aligned}
& =\int_{-\infty}^{+\infty}\left|\frac{\partial}{\partial x}\left(\sigma_{2}(s, x) A\left(s, x_{1}, x\right) \frac{\partial}{\partial x} F_{2}(s, x)\right)\right| d x \\
& =\int_{-\infty}^{+\infty}\left|\frac{\partial}{\partial x}\left(\sigma_{2}(s, x) A\left(s, x_{1}, x\right)\right) \frac{\partial}{\partial x} F_{2}(s, x)+\sigma(s, x) A\left(s, x_{1}, x\right) \frac{\partial^{2}}{\partial x^{2}} F_{2}(s, x)\right| d x \\
& \leq \int_{-\infty}^{+\infty} L_{A} \frac{\partial}{\partial x} F_{2}(s, x) d x+\int_{-\infty}^{+\infty}\left(m+L_{A}|x|\right)\left|\frac{\partial^{2}}{\partial x^{2}} F_{2}(s, x)\right| d x \\
& \leq \quad L_{A}+\int_{-\infty}^{+\infty}\left(m+L_{A}|x|\right) \frac{M}{1+|x|^{2+\varepsilon}} d x<\infty
\end{aligned}
$$

Now we switch to the bivariate case.

Theorem 6.1. Let

$$
h: \mathbb{R}^{2} \longrightarrow \mathbb{R}
$$

be an infinitely many times differentiable function with a compact support. Then for any $t>0$

$$
\begin{aligned}
& \int_{[0,1]^{2}} h\left(v_{1}, v_{2}\right) C_{t}\left(v_{1}, v_{2}\right) d v_{1} d v_{2} \\
& \quad=\int_{[0,1]^{2}} h\left(v_{1}, v_{2}\right) C_{1}\left(v_{1}, v_{2}\right) d v_{1} d v_{2}-\int_{0}^{t} B_{s}\left(h, C_{s}\right) d s,
\end{aligned}
$$

where for $t \geq 0$ and a copula $C$

$$
\begin{aligned}
B_{t}(h, C) & =\frac{1}{2} \int_{[0,1]^{2}} \frac{\partial}{\partial v_{1}}\left(h\left(v_{1}, v_{2}\right) v_{1,2}\left(t, v_{1}\right)^{2}\right) D_{1} C\left(v_{1}, v_{2}\right) d v_{1} d v_{2} \\
& +\frac{1}{2} \int_{[0,1]^{2}} \frac{\partial}{\partial v_{2}}\left(h\left(v_{1}, v_{2}\right) v_{2,2}\left(t, v_{2}\right)^{2}\right) D_{2} C\left(v_{1}, v_{2}\right) d v_{1} d v_{2} \\
& +\int_{[0,1]^{2}} \frac{\partial}{\partial v_{2}}\left(h\left(v_{1}, v_{2}\right) v_{1,2}\left(t, v_{1}\right) v_{2,2}\left(t, v_{2}\right) A_{c}\left(t, v_{1}, v_{2}\right)\right) D_{1} C\left(v_{1}, v_{2}\right) d v_{1} d v_{2}
\end{aligned}
$$

Proof.

We recall Ito's formula. Let

$$
H: \mathbb{R}^{2} \longrightarrow \mathbb{R}
$$

be a twice continuously differentiable function. Then for $t>0$

$$
\begin{aligned}
E\left(H\left(U_{1, t}, U_{2, t}\right)\right) & =E\left(H\left(U_{1,1}, U_{2,1}\right)\right. \\
& +\int_{0}^{t} E\left(\sum_{j=1}^{2} \frac{\partial H}{\partial u_{j}}\left(U_{1, s}, U_{2, s}\right) v_{j, 1}\left(s, U_{j, s}\right)\right. \\
& +\frac{1}{2} \sum_{j=1}^{2} \frac{\partial^{2} H}{\partial u_{j}^{2}}\left(U_{1, s}, U_{2, s}\right) v_{j, 2}\left(s, U_{j, s}\right)^{2} \\
& \left.+\frac{\partial^{2} H}{\partial u_{1} \partial u_{2}}\left(U_{1, s}, U_{2, s}\right) v_{1,2}\left(s, U_{1, s}\right) v_{2,2}\left(s, U_{2, s}\right) A_{C}\left(s, U_{1, s}, U_{2, s}\right)\right) d s .
\end{aligned}
$$


Applying an operator like approach, we can abbreviate the above as

$$
\begin{aligned}
E\left(H\left(U_{1, t}, U_{2, t}\right)\right) & -E\left(H\left(U_{1,1}, U_{2,1}\right)\right) \\
& =\int_{0}^{t} E\left(\sum_{(i, j) \in I} \frac{\partial^{i+j} H}{\partial u_{1}^{i} \partial u_{2}^{j}}\left(U_{1, s}, U_{2, s}\right) L_{i, j}\left(s, U_{1, s}, U_{2, s}\right)\right) d s,
\end{aligned}
$$

where the set $I$ consists of 5 pairs of indices

$$
I=\{(0,1),(0,2),(1,0),(2,0),(1,1)\} .
$$

Next we substitute as $H$ a convolution of an infinitely many times differentiable function with compact support $h$ and an indicator function $\kappa\left(u_{1}\right) \kappa\left(u_{2}\right)$

$$
\begin{gathered}
\kappa(u)= \begin{cases}1 & \text { when } u \leq 0, \\
0 & \text { otherwise. }\end{cases} \\
H\left(u_{1}, u_{2}\right)=\int_{\mathbb{R}^{2}} h\left(v_{1}, v_{2}\right) \kappa\left(u_{1}-v_{1}\right) \kappa\left(u_{2}-v_{2}\right) d v_{1} d v_{2} .
\end{gathered}
$$

Note that $H$ is infinitely many times differentiable.

$$
\begin{aligned}
\frac{\partial H}{\partial u_{j}}(u) & =\int_{\mathbb{R}^{2}} \frac{\partial h}{\partial v_{j}}\left(v_{1}, v_{2}\right) \kappa\left(u_{1}-v_{1}\right) \kappa\left(u_{2}-v_{2}\right) d v_{1} d v_{2}, \\
\frac{\partial^{2} H}{\partial u_{j} \partial u_{k}}(u) & =\int_{\mathbb{R}^{2}} \frac{\partial^{2} h}{\partial v_{j} \partial v_{k}}\left(v_{1}, v_{2}\right) \kappa\left(u_{1}-v_{1}\right) \kappa\left(u_{2}-v_{2}\right) d v_{1} d v_{2} .
\end{aligned}
$$

Furthermore note that for every $u=\left(u_{1}, u_{2}\right)$

$$
\begin{aligned}
\left|H\left(u_{1}, u_{2}\right)\right| & \leq \int_{\mathbb{R}^{2}}\left|h\left(v_{1}, v_{2}\right)\right| \kappa\left(u_{1}-v_{1}\right) \kappa\left(u_{2}-v_{2}\right) d v_{1} d v_{2} \\
& \leq \int_{\mathbb{R}^{2}}\left|h\left(v_{1}, v_{2}\right)\right| d v_{1} d v_{2}=|| h \|_{L^{1}} .
\end{aligned}
$$

Replacing $h$ by its derivative we get

$$
\left|\frac{\partial H}{\partial u_{j}}(u)\right| \leq|| \frac{\partial h}{\partial v_{j}}||_{L^{1}}
$$

and

$$
\left|\frac{\partial^{2} H}{\partial u_{j} \partial u_{k}}(u)\right| \leq\left\|\frac{\partial^{2} h}{\partial v_{j} \partial v_{k}}\right\|_{L^{1}} .
$$

Using the Fubini Theorem, Sklar Theorem and the boundary properties of copulas we get for $t \geq 0$

$$
\begin{aligned}
& E\left(H\left(U_{1, t}, U_{2, t}\right)\right)-E\left(H\left(U_{1,1}, U_{2,1}\right)\right) \\
= & E \int_{\mathbb{R}^{2}} h\left(v_{1}, v_{2}\right)\left(\kappa\left(U_{1, t}-v_{1}\right) \kappa\left(U_{2, t}-v_{2}\right)-\kappa\left(U_{1,1}-v_{1}\right) \kappa\left(U_{2,1}-v_{2}\right)\right) d v_{1} d v_{2} \\
\stackrel{F}{=} & \int_{\mathbb{R}^{2}} h\left(v_{1}, v_{2}\right) E\left(\kappa\left(U_{1, t}-v_{1}\right) \kappa\left(U_{2, t}-v_{2}\right)-\kappa\left(U_{1,1}-v_{1}\right) \kappa\left(U_{2,1}-v_{2}\right)\right) d v_{1} d v_{2} \\
\stackrel{S}{=} & \int_{\mathbb{R}^{2}} h\left(v_{1}, v_{2}\right)\left(C_{t}\left(\min \left(1, v_{1}^{+}\right), \min \left(1, v_{2}^{+}\right)\right)-C_{1}\left(\min \left(1, v_{1}^{+}\right), \min \left(1, v_{2}^{+}\right)\right)\right) d v_{1} d v_{2} \\
& \int_{[0,1]^{2}} h\left(v_{1}, v_{2}\right)\left(C_{t}\left(v_{1}, v_{2}\right)-C_{1}\left(v_{1}, v_{2}\right)\right) d v_{1} d v_{2} .
\end{aligned}
$$


Let $B_{s}$ denote the integrand in formulas (46) and (47) multiplied by -1 . Since $P\left(U_{j, s} \leq 0\right)=0$, in a similar way as above, we obtain for $s \geq 0$

$$
\begin{aligned}
-B_{S} & =E\left(\sum_{(i, j) \in I} \frac{\partial^{i+j} H}{\partial u_{1}^{i} \partial u_{2}^{j}}\left(U_{1, s}, U_{2, s}\right) L_{i, j}\left(s, U_{1, s}, U_{2, s}\right)\right) \\
& =\sum_{(i, j) \in I} E\left(\int_{\mathbb{R}^{2}} \frac{\partial^{i+j} h\left(v_{1}, v_{2}\right)}{\partial v_{1}^{i} \partial v_{2}^{j}} \kappa\left(U_{1, s}-v_{1}\right) \kappa\left(U_{2, s}-v_{2}\right) L_{i, j}\left(s, U_{1, s}, U_{2, s}\right) d v_{1} d v_{2}\right) \\
& \underline{F} \sum_{(i, j) \in I_{\mathbb{R}^{2}}} \int_{\frac{\partial^{i+j} h\left(v_{1}, v_{2}\right)}{\partial v_{1}^{i} \partial v_{2}^{j}} E\left(\kappa\left(U_{1, s}-v_{1}\right) \kappa\left(U_{2, s}-v_{2}\right) L_{i, j}\left(s, U_{1, s}, U_{2, s}\right)\right) d v_{1} d v_{2}} \\
& =\sum_{\left.(i, j) \in I_{[0,+\infty}\right)^{2}} \frac{\partial^{i+j} h\left(v_{1}, v_{2}\right)}{\partial v_{1}^{i} \partial v_{2}^{j}} E\left(\kappa\left(U_{1, s}-v_{1}\right) \kappa\left(U_{2, s}-v_{2}\right) L_{i, j}\left(s, U_{1, s}, U_{2, s}\right)\right) d v_{1} d v_{2} .
\end{aligned}
$$

Now we apply the chain rule for conditional expectations

$$
E\left(F\left(U_{1}, U_{2}\right)\right)=E\left(E\left(F\left(U_{1}, U_{2}\right) \mid U_{j}\right)\right)=\int_{0}^{1} E\left(F\left(U_{1}, U_{2}\right) \mid U_{j}=\xi\right) d \xi
$$

and obtain

$$
\begin{aligned}
-B_{s}= & \sum_{i=1}^{2} \int_{[0,+\infty)^{2}} \frac{\partial^{i} h\left(v_{1}, v_{2}\right)}{\partial v_{1}^{i}} \\
& \times \int_{0}^{\min \left(1, v_{1}\right)} E\left(\kappa\left(U_{2, s}-v_{2}\right) L_{i, 0}\left(s, \xi, U_{2, s}\right) \mid U_{1, s}=\xi\right) d \xi d v_{1} d v_{2} \\
& +\sum_{j=1}^{2} \int_{[0,+\infty)^{2}} \frac{\partial^{j} h\left(v_{1}, v_{2}\right)}{\partial v_{2}^{j}} \\
& \times \int_{0}^{\min \left(1, v_{2}\right)} E\left(\kappa\left(U_{1, s}-v_{1}\right) L_{0, j}\left(s, U_{1, s}, \xi\right) \mid U_{2, s}=\xi\right) d \xi d v_{1} d v_{2} \\
& \int_{[0,+\infty)^{2}}^{\min \left(1, v_{1}\right)} \frac{\partial^{2} h\left(v_{1}, v_{2}\right)}{\partial v_{1} \partial v_{2}} \\
& \int_{0} E\left(\kappa\left(U_{2, s}-v_{2}\right) L_{1,1}\left(s, \xi, U_{2, s}\right) \mid U_{1, s}=\xi\right) d \xi d v_{1} d v_{2} .
\end{aligned}
$$

Next we integrate by parts.

$$
\begin{aligned}
B_{s} & \stackrel{I P}{=} \sum_{i=0}^{1} \int_{0}^{+\infty} \int_{0}^{1} \frac{\partial^{i} h\left(v_{1}, v_{2}\right)}{\partial v_{1}^{i}} E\left(\kappa\left(U_{2, s}-v_{2}\right) L_{i+1,0}\left(s, v_{1}, U_{2, s}\right) \mid U_{1, s}=v_{1}\right) d v_{1} d v_{2} \\
& +\sum_{j=0}^{1} \int_{0}^{+\infty} \int_{0}^{1} \frac{\partial^{j} h\left(v_{1}, v_{2}\right)}{\partial v_{2}^{j}} E\left(\kappa\left(U_{1, s}-v_{1}\right) L_{0, j+1}\left(s, U_{1, s}, v_{2}\right) \mid U_{2, s}=v_{2}\right) d v_{2} d v_{1} \\
& +\int_{0}^{+\infty} \int_{0}^{1} \frac{\partial h\left(v_{1}, v_{2}\right)}{\partial v_{2}} E\left(\kappa\left(U_{2, s}-v_{2}\right) L_{1,1}\left(s, v_{1}, U_{2, s}\right) \mid U_{1, s}=v_{1}\right) d v_{1} d v_{2}
\end{aligned}
$$


Setting

$$
L_{1,0}=v_{1,1}, \quad L_{0,1}=v_{2,1}, \quad L_{2,0}=\frac{1}{2} v_{1,2}^{2}, \quad L_{0,2}=\frac{1}{2} v_{2,2}^{2}, \quad L_{1,1}=v_{1,2} v_{2,2} A_{C},
$$

we get

$$
\begin{aligned}
& B_{s}=\int_{0}^{+\infty} \int_{0}^{1}\left(h\left(v_{1}, v_{2}\right) v_{1,1}\left(s, v_{1}\right)+\frac{1}{2} \frac{\partial h\left(v_{1}, v_{2}\right)}{\partial v_{1}} v_{1,2}\left(s, v_{1}\right)^{2}\right) \\
& \left.\times E\left(\kappa\left(U_{2, s}-v_{2}\right)\right) \mid U_{1, s}=v_{1}\right) d v_{1} d v_{2} \\
& +\int_{0}^{1} \int_{0}^{+\infty}\left(h\left(v_{1}, v_{2}\right) v_{2,1}\left(s, v_{2}\right)+\frac{1}{2} \frac{\partial h\left(v_{1}, v_{2}\right)}{\partial v_{2}} v_{2,2}\left(s, v_{2}\right)^{2}\right) \\
& \left.\quad \times E\left(\kappa\left(U_{1, s}-v_{1}\right)\right) \mid U_{2, s}=v_{2}\right) d v_{1} d v_{2} \\
& +\int_{0}^{+\infty} \int_{0}^{1} \frac{\partial h\left(v_{1}, v_{2}\right)}{\partial v_{2}} v_{1,2}\left(s, v_{1}\right) \\
& \quad \times E\left(\kappa\left(U_{2, s}-v_{2}\right) v_{2,2}\left(s, U_{2, s}\right) A_{C}\left(s, v_{1}, U_{2, s}\right) \mid U_{1, s}=v_{1}\right) d v_{1} d v_{2} .
\end{aligned}
$$

We apply the Fubini Theorem to the last summand and recall that $v_{j, 1}$ are the derivatives of $v_{j, 2}^{2} / 2$ (Proposition 3.1).

$$
\begin{aligned}
& B_{S}=\int_{0}^{+\infty} \int_{0}^{1}\left(h\left(v_{1}, v_{2}\right) \frac{1}{2} \frac{\partial v_{1,2}\left(s, v_{1}\right)^{2}}{\partial v_{1}}+\frac{1}{2} \frac{\partial h\left(v_{1}, v_{2}\right)}{\partial v_{1}} v_{1,2}\left(s, v_{1}\right)^{2}\right) \\
& \left.\times E\left(\kappa\left(U_{2, s}-v_{2}\right)\right) \mid U_{1, s}=v_{1}\right) d v_{1} d v_{2} \\
& +\int_{0}^{1} \int_{0}^{+\infty}\left(h\left(v_{1}, v_{2}\right) \frac{1}{2} \frac{\partial v_{2,2}\left(s, v_{2}\right)^{2}}{\partial v_{2}}+\frac{1}{2} \frac{\partial h\left(v_{1}, v_{2}\right)}{\partial v_{2}} v_{2,2}\left(s, v_{2}\right)^{2}\right) \\
& \left.\quad \times E\left(\kappa\left(U_{1, s}-v_{1}\right)\right) \mid U_{2, s}=v_{2}\right) d v_{1} d v_{2} \\
& +\int_{0}^{1} v_{1,2}\left(s, v_{1}\right) \\
& \left.\times E\left(\int_{0}^{+\infty} \kappa\left(U_{2, s}-v_{2}\right) \frac{\partial h\left(v_{1}, v_{2}\right)}{\partial v_{2}} d v_{2}\right) v_{2,2}\left(s, U_{2, s}\right) A_{C}\left(s, v_{1}, U_{2, s}\right) \mid U_{1, s}=v_{1}\right) d v_{1} \\
& \left.=\frac{1}{2} \int_{0}^{+\infty} \int_{0}^{1} \frac{\partial}{\partial v_{1}}\left(h\left(v_{1}, v_{2}\right) v_{1,2}\left(s, v_{1}\right)^{2}\right) E\left(\kappa\left(U_{2, s}-v_{2}\right)\right) \mid U_{1, s}=v_{1}\right) d v_{1} d v_{2} \\
& \left.+\frac{1}{2} \int_{0}^{1} \int_{0}^{+\infty} \frac{\partial}{\partial v_{2}}\left(h\left(v_{1}, v_{2}\right) v_{2,2}\left(s, v_{2}\right)^{2}\right) E\left(\kappa\left(U_{1, s}-v_{1}\right)\right) \mid U_{2, s}=v_{2}\right) d v_{1} d v_{2} \\
& +\int_{0}^{1} v_{1,2}\left(s, v_{1}\right) E\left(-h\left(v_{1}, U_{2, s}\right) v_{2,2}\left(s, U_{2, s}\right) A_{C}\left(s, v_{1}, U_{2, s}\right) \mid U_{1, s}=v_{1}\right) d v_{1}
\end{aligned}
$$

Now we show that the domain of integration can be restricted to the unit square. Since for $x \geq 1$ and $s \geq 0$

$$
\kappa\left(U_{2, s}-x\right) \stackrel{a s}{=} \kappa\left(U_{1, s}-x\right) \stackrel{a s}{=} 1,
$$

and $v_{i, 2}$ are vanishing at the ends of the unit interval we have

$$
\left.\frac{1}{2} \int_{1}^{+\infty} \int_{0}^{1} \frac{\partial}{\partial v_{1}}\left(h\left(v_{1}, v_{2}\right) v_{1,2}\left(s, v_{1}\right)^{2}\right) E\left(\kappa\left(U_{2, s}-v_{2}\right)\right) \mid U_{1, s}=v_{1}\right) d v_{1} d v_{2}
$$




$$
\begin{aligned}
& =\frac{1}{2} \int_{1}^{+\infty} \int_{0}^{1} \frac{\partial}{\partial v_{1}}\left(h\left(v_{1}, v_{2}\right) v_{1,2}\left(s, v_{1}\right)^{2}\right) d v_{1} d v_{2} \\
& =\left.\int_{1}^{+\infty} h\left(v_{1}, v_{2}\right) \partial v_{1,2}\left(s, v_{1}\right)^{2}\right|_{v_{1}=0} ^{v_{1}=1} d v_{2}=0
\end{aligned}
$$

In a similar way we obtain

$$
\left.\frac{1}{2} \int_{0}^{1} \int_{1}^{+\infty} \frac{\partial}{\partial v_{2}}\left(h\left(v_{1}, v_{2}\right) v_{2,2}\left(s, v_{2}\right)^{2}\right) E\left(\kappa\left(U_{1, s}-v_{1}\right)\right) \mid U_{2, s}=v_{2}\right) d v_{1} d v_{2}=0
$$

To finish the proof we observe that for $v_{1}, v_{2} \in[0,1]$

$$
\begin{array}{lll}
\left.E\left(\kappa\left(U_{2, s}-v_{2}\right)\right) \mid U_{1, s}=v_{1}\right) & \stackrel{a e}{=} & D_{1} C_{s}\left(v_{1}, v_{2}\right), \\
\left.E\left(\kappa\left(U_{1, s}-v_{1}\right)\right) \mid U_{2, s}=v_{2}\right) & \stackrel{a e}{=} & D_{2} C_{s}\left(v_{1}, v_{2}\right) .
\end{array}
$$

Furthermore due to Lemma 6.3 we may apply once more the Fubini Theorem.

$$
\begin{aligned}
& E\left(-h\left(v_{1}, U_{2, s}\right) v_{2,2}\left(s, U_{2, s}\right) A_{C}\left(s, v_{1}, U_{2, s}\right) \mid U_{1, s}=v_{1}\right) \\
= & E\left(\int_{U_{2, s}}^{1} \frac{\partial}{\partial v_{2}}\left(h\left(v_{1}, v_{2}\right) v_{2,2}\left(s, v_{2}\right) A_{C}\left(s, v_{1}, v_{2}\right)\right) d v_{2} \mid U_{1, s}=v_{1}\right) \\
= & E\left(\int_{0}^{1} \kappa\left(U_{2, s}-v_{2}\right) \frac{\partial}{\partial v_{2}}\left(h\left(v_{1}, v_{2}\right) v_{2,2}\left(s, v_{2}\right) A_{C}\left(s, v_{1}, v_{2}\right)\right) d v_{2} \mid U_{1, s}=v_{1}\right) \\
= & \int_{0}^{1} \frac{\partial}{\partial v_{2}}\left(h\left(v_{1}, v_{2}\right) v_{2,2}\left(s, v_{2}\right) A_{C}\left(s, v_{1}, v_{2}\right)\right) E\left(\kappa\left(U_{2, s}-v_{2}\right) \mid U_{1, s}=v_{1}\right) d v_{2} \\
= & \int_{0}^{1} \frac{\partial}{\partial v_{2}}\left(h\left(v_{1}, v_{2}\right) v_{2,2}\left(s, v_{2}\right) A_{C}\left(s, v_{1}, v_{2}\right)\right) D_{1} C_{s}\left(v_{1}, v_{2}\right) d v_{2}
\end{aligned}
$$

Applying the above to (56) we get

$$
\begin{aligned}
B_{s} & =\frac{1}{2} \int_{[0,1]^{2}} \frac{\partial}{\partial v_{1}}\left(h\left(v_{1}, v_{2}\right) v_{1,2}\left(s, v_{1}\right)^{2}\right) D_{1} C_{s}\left(v_{1}, v_{2}\right) d v_{1} d v_{2} \\
& +\frac{1}{2} \int_{[0,1]^{2}} \frac{\partial}{\partial v_{2}}\left(h\left(v_{1}, v_{2}\right) v_{2,2}\left(s, v_{2}\right)^{2}\right) D_{2} C_{s}\left(v_{1}, v_{2}\right) d v_{1} d v_{2} \\
& +\int_{[0,1]^{2}} \frac{\partial}{\partial v_{2}}\left(h\left(v_{1}, v_{2}\right) v_{1,2}\left(s, v_{1}\right) v_{2,2}\left(s, v_{2}\right) A_{c}\left(s, v_{1}, v_{2}\right)\right) D_{1} C_{s}\left(v_{1}, v_{2}\right) d v_{1} d v_{2}
\end{aligned}
$$

We extend the bilinear form $B_{t}$ for Sobolev test functions. We set for $h \in H^{1}$

$$
\begin{aligned}
B_{t}(h, C) & =\frac{1}{2} \int_{[0,1]^{2}} D_{1}\left(h\left(v_{1}, v_{2}\right) v_{1,2}\left(t, v_{1}\right)^{2}\right) D_{1} C\left(v_{1}, v_{2}\right) d v_{1} d v_{2} \\
& +\frac{1}{2} \int_{[0,1]^{2}} D_{2}\left(h\left(v_{1}, v_{2}\right) v_{2,2}\left(t, v_{2}\right)^{2}\right) D_{2} C\left(v_{1}, v_{2}\right) d v_{1} d v_{2}
\end{aligned}
$$




$$
+\int_{[0,1]^{2}} D_{2}\left(h\left(v_{1}, v_{2}\right) v_{1,2}\left(t, v_{1}\right) v_{2,2}\left(t, v_{2}\right) A_{c}\left(t, v_{1}, v_{2}\right)\right) D_{1} C\left(v_{1}, v_{2}\right) d v_{1} d v_{2}
$$

We recall two useful facts about Sobolev functions from $H^{1}$.

We start with absolutely continuity on lines (ACL) of Sobolev functions (see for example [19] §1.1.3.).

If a function is in $H^{1}\left(\mathbb{R}^{2}\right)$, then, possibly after modifying the function on a set of measure zero, the restriction to almost every line parallel to the coordinate directions in $\mathbb{R}^{2}$ is absolutely continuous; what is more, the classical derivative along the lines that are parallel to the coordinate directions are in $L^{2}$.

Therefore when $h \in H^{1}\left(\mathbb{R}^{2}\right)$ has a compact support, then

$$
\begin{aligned}
h(x, y) & \stackrel{a e}{=} \int_{-\infty}^{x} D_{1} h(\xi, y) d \xi=\int_{-\infty}^{+\infty} \kappa(\xi-x) D_{1} h(\xi, y) d \xi \\
& =-\int_{x}^{+\infty} D_{1} h(\xi, y) d \xi=-\int_{-\infty}^{+\infty} \kappa(x-\xi) D_{1} h(\xi, y) d \xi
\end{aligned}
$$

Similarly

$$
\begin{aligned}
h(x, y) & \stackrel{a e}{=} \int_{-\infty}^{y} D_{2} h(x, \xi) d \xi=\int_{-\infty}^{+\infty} \kappa(\xi-y) D_{2} h(x, \xi) d \xi \\
& =-\int_{y}^{+\infty} D_{2} h(x, \xi) d \xi=-\int_{-\infty}^{+\infty} \kappa(y-\xi) D_{2} h(x, \xi) d \xi .
\end{aligned}
$$

The second property is the possibility of approximation of Sobolev functions with compact supports by smooth functions with compact supports as well. We fix a cut-off function $\Psi$.

$$
\Psi: \mathbb{R} \longrightarrow \mathbb{R}, \quad \Psi(x)=\left\{\begin{array}{ccc}
1 & \text { for } & |x| \leq 1, \\
2-|x| & \text { for } & 1<|x| \leq 2, \\
0 & \text { for } & 2<|x| .
\end{array}\right.
$$

Let $h$ be a Sobolev function from $H^{1}\left(\mathbb{R}^{2}\right)$. To "bound" the support, we set

$$
h_{b}\left(v_{1}, v_{2}\right)=h\left(v_{1}, v_{2}\right) \Psi\left(v_{1}\right) \Psi\left(v_{2}\right) .
$$

Then there exists a sequence of $C^{\infty}$ functions with compact supports $h_{k}, k=1,2, \ldots$ which approximate $h_{b}\left(v_{1}, v_{2}\right)$ in Sobolev norm $\|\cdot\|_{H^{1}}$ (compare [9] §5.3, [19] §1.1.5.).

$$
\lim _{k \rightarrow \infty}\left\|h_{b}\left(v_{1}, v_{2}\right)-h_{k}\left(v_{1}, v_{2}\right)\right\|_{H^{1}}=0 .
$$

Basing on the above, we show that the extended bilinear form $B_{t}$ for fixed copula $C$ is a bounded linear mapping of test functions $h$.

Lemma 6.4. We fix $T>0$. For any test function $h \in H^{1}$, any copula $C$ and $s \in[0, T]$ we have

$$
\left|B_{S}(h, C)\right| \leq L_{T}|| h \|_{H^{1}}
$$

where

$$
\begin{aligned}
L_{T}= & \max \left\{\left|v_{1,1}\left(s, v_{1}\right)\right|+\left|v_{2,1}\left(s, v_{2}\right)\right|+\left(\left|v_{2,1}\left(s, v_{3}\right)\right|+\left|v_{2,2}\left(s, v_{4}\right)\right|\right)^{2}\right. \\
& \left.: s \in[1, T],\left(v_{1}, v_{2}, v_{3}, v_{4}\right) \in[0,1]^{4}\right\}
\end{aligned}
$$


Proof.

We start with the following estimate:

$$
\begin{aligned}
\left|B_{t}(h, C)\right| & \leq \frac{1}{2}\left|\int_{[0,1]^{2}} D_{1}\left(h\left(v_{1}, v_{2}\right) v_{1,2}\left(t, v_{1}\right)^{2}\right) D_{1} C\left(v_{1}, v_{2}\right) d v_{1} d v_{2}\right| \\
& +\left|\frac{1}{2} \int_{[0,1]^{2}} D_{2}\left(h\left(v_{1}, v_{2}\right) v_{2,2}\left(t, v_{2}\right)^{2}\right) D_{2} C\left(v_{1}, v_{2}\right) d v_{1} d v_{2}\right| \\
& +\left|\int_{[0,1]^{2}} D_{2}\left(h\left(v_{1}, v_{2}\right) v_{1,2}\left(t, v_{1}\right) v_{2,2}\left(t, v_{2}\right) A_{C}\left(t, v_{1}, v_{2}\right)\right) D_{1} C\left(v_{1}, v_{2}\right) d v_{1} d v_{2}\right|
\end{aligned}
$$

Since copulas are nondecreasing Lipschitz functions with Lipschitz constant $1, D_{i} C\left(v_{1}, v_{2}\right) \in[0,1]$. Hence due to Hölder-Schwarz inequality ([9] Appendix B2)

$$
\begin{aligned}
& \left|\int_{[0,1]^{2}} D_{1}\left(h\left(v_{1}, v_{2}\right) v_{1,2}\left(t, v_{1}\right)^{2}\right) D_{1} C\left(v_{1}, v_{2}\right) d v_{1} d v_{2}\right| \\
& \leq \int_{[0,1]^{2}}\left|D_{1}\left(h\left(v_{1}, v_{2}\right) v_{1,2}\left(t, v_{1}\right)^{2}\right)\right| d v_{1} d v_{2} \\
& \leq \int_{[0,1]^{2}}\left(\left|D_{1} h\left(v_{1}, v_{2}\right) v_{1,2}\left(t, v_{1}\right)^{2}\right|+2\left|h\left(v_{1}, v_{2}\right) v_{1,1}\left(t, v_{1}\right)\right|\right) d v_{1} d v_{2} \\
& \leq\left\|D_{1} h\right\|_{L^{2}} \cdot\left\|v_{1,2}^{2}(t, \cdot)\right\|_{L^{2}}+2|| D_{1} h\left\|_{L^{2}} \cdot\right\| v_{1,1}(t, \cdot) \|_{L^{2}} \\
& \leq\|h\|_{H^{1}\left(\mathbb{R}^{2}\right)} \cdot\left(\left\|v_{1,2}^{2}(t, \cdot)\right\|_{L^{2}}+2\left\|v_{1,1}(t, \cdot)\right\|_{L^{2}}\right) . \\
& \leq\|h\|_{H^{1}\left(\mathbb{R}^{2}\right)} \cdot\left(\left\|v_{1,2}^{2}(t, \cdot)\right\|_{L^{\infty}}+2\left\|v_{1,1}(t, \cdot)\right\|_{L^{\infty}}\right) .
\end{aligned}
$$

In a similar way we get

$$
\begin{aligned}
& \left|\int_{0,1]^{2}} D_{2}\left(h\left(v_{1}, v_{2}\right) v_{2,2}\left(t, v_{2}\right)^{2}\right) D_{2} C\left(v_{1}, v_{2}\right) d v_{1} d v_{2}\right| \\
& \leq\|h\|_{H^{1}\left(\mathbb{R}^{2}\right)} \cdot\left(\left\|v_{2,2}^{2}(t, \cdot)\right\|_{L^{\infty}}+2\left\|v_{2,1}(t, \cdot)\right\|_{L^{\infty}}\right) .
\end{aligned}
$$

The estimation of the last summand is more complicated.

We invert the transformations from the proof of Theorem 6.1 and then apply Hölder-Schwarz inequality. Let $U_{1}, U_{2}$ be representers of the copula $C$ and $\Psi$ be the cut-off function defined by formula (65).

$$
\begin{aligned}
& \left|\int_{0,1]^{2}} D_{2}\left(h\left(v_{1}, v_{2}\right) v_{1,2}\left(t, v_{1}\right) v_{2,2}\left(t, v_{2}\right) A_{c}\left(t, v_{1}, v_{2}\right)\right) D_{1} C\left(v_{1}, v_{2}\right) d v_{1} d v_{2}\right| \\
= & \left|\int_{\mid 0,1]^{2}} D_{2}\left(h\left(v_{1}, v_{2}\right) \Psi\left(v_{2}\right) v_{1,2}\left(t, v_{1}\right) v_{2,2}\left(t, v_{2}\right) A_{c}\left(t, v_{1}, v_{2}\right)\right) D_{1} C\left(v_{1}, v_{2}\right) d v_{1} d v_{2}\right| \\
= & \left.\left|\int_{0,1]^{2}} D_{2}\left(h\left(v_{1}, v_{2}\right) \Psi\left(v_{2}\right) v_{1,2}\left(t, v_{1}\right) v_{2,2}\left(t, v_{2}\right) A_{c}\left(t, v_{1}, v_{2}\right)\right) E\left(\kappa\left(U_{2}-v_{2}\right)\right)\right| U_{1}=v_{1}\right) d v_{2} d v_{1} \mid \\
= & \left|\int_{[0,1]} E\left(h\left(v_{1}, U_{2}\right) \Psi\left(U_{2}\right) v_{1,2}\left(t, v_{1}\right) v_{2,2}\left(t, U_{2}\right) A_{c}\left(t, v_{1}, U_{2}\right) \mid U_{1}=v_{1}\right) d v_{1}\right|
\end{aligned}
$$




$$
\begin{aligned}
& =\left|\int_{0}^{1} E\left(\int_{0}^{2} \kappa\left(U_{2}-v\right) D_{2}\left(h\left(v_{1}, v\right) \Psi(v)\right) v_{1,2}\left(t, v_{1}\right) v_{2,2}\left(t, U_{2}\right) A_{c}\left(t, v_{1}, U_{2}\right) d v \mid U_{1}=v_{1}\right) d v_{1}\right| \\
& \underline{F}\left|\int_{0}^{1} \int_{0}^{2} E\left(\kappa\left(U_{2}-v\right) D_{2}\left(h\left(v_{1}, v\right) \Psi(v)\right) v_{1,2}\left(t, v_{1}\right) v_{2,2}\left(t, U_{2}\right) A_{c}\left(t, v_{1}, U_{2}\right) \mid U_{1}=v_{1}\right) d v d v_{1}\right| \\
& =\left|\int_{0}^{1} \int_{0}^{2} D_{2}\left(h\left(v_{1}, v\right) \Psi(v)\right) v_{1,2}\left(t, v_{1}\right) E\left(\kappa\left(U_{2}-v\right) v_{2,2}\left(t, U_{2}\right) A_{c}\left(t, v_{1}, U_{2}\right) \mid U_{1}=v_{1}\right) d v d v_{1}\right| \\
& \leq\left\|v_{1,2}(t, \cdot)\right\|_{L^{\infty}}\left\|v_{2,2}(t, \cdot)\right\|_{L^{\infty}} \int_{0}^{1} \int_{0}^{2}\left(\left|D_{2} h\left(v_{1}, v\right)\right| \Psi(v)+\left|h\left(v_{1}, v\right) D \Psi(v)\right|\right) d v d v_{1} \\
& \leq\left\|v_{1,2}(t, \cdot)\right\|_{L^{\infty}}\left\|v_{2,2}(t, \cdot)\right\|_{L^{\infty}}\left(\frac{2 \sqrt{3}}{3}\left\|D_{2} h\right\|_{L^{2}}+\frac{1}{2}\|h\|_{L^{2}}\right) \\
& \leq 2\left\|v_{1,2}(t, \cdot)\right\|_{L^{\infty}}\left\|v_{2,2}(t, \cdot)\right\|_{L^{\infty}}\|h\|_{H^{1}\left(\mathbb{R}^{2}\right)}
\end{aligned}
$$

Putting the above three estimates together we obtain

$$
\begin{aligned}
\left|B_{t}(h, C)\right| \leq & \|h\|_{H^{1}\left(\mathbb{R}^{2}\right)} \cdot\left(\left\|v_{1,1}(t, \cdot)\right\|_{L^{\infty}}+\left\|v_{2,1}(t, \cdot)\right\|_{L^{\infty}}\right. \\
& \left.+\left\|v_{1,2}^{2}(t, \cdot)\right\|_{L^{\infty}}+\left\|v_{2,2}^{2}(t, \cdot)\right\|_{L^{\infty}}+2\left\|v_{1,2}(t, \cdot)\right\|\left\|_{L^{\infty}}\right\| v_{2,2}(t, \cdot) \|_{L^{\infty}}\right) \\
\leq & \|h\|_{H^{1}\left(\mathbb{R}^{2}\right)} \cdot\left(\left\|v_{1,1}(t, \cdot)\right\|_{L^{\infty}}+v_{2,1}(t, \cdot) \|_{L^{\infty}}+\left(\left\|v_{1,2}(t, \cdot)\right\|_{L^{\infty}}+\left\|v_{2,2}(t, \cdot)\right\|_{L^{\infty}}\right)^{2}\right)
\end{aligned}
$$

This implies the following uniform bound. For all $t \in[0, T]$ we have

$$
\left|B_{t}(h, C)\right| \leq L_{T}|| h \|_{H^{1}\left(\mathbb{R}^{2}\right)},
$$

where

$$
\begin{aligned}
L_{T}= & \max \left\{\left|v_{1,1}\left(s, v_{1}\right)\right|+\left|v_{2,1}\left(s, v_{2}\right)\right|+\left(\left|v_{2,1}\left(s, v_{3}\right)\right|+\left|v_{2,2}\left(s, v_{4}\right)\right|\right)^{2}\right. \\
& \left.: s \in[1, T],\left(v_{1}, v_{2}, v_{3}, v_{4}\right) \in[0,1]^{4}\right\} .
\end{aligned}
$$

Since $v_{i, j}(t, v)$ are continuous functions the bound $L_{T}$ is well-defined.

Since $B_{S}(\cdot, C)$ are uniformly bounded linear functionals on $H^{1}$, Theorem 6.1 remains valid for Sobolev test functions.

Corollary 6.1. Let $h \in H^{1}\left(\mathbb{R}^{2}\right)$. Then for any $t>0$

$$
\begin{aligned}
& \int_{[0,1]^{2}} h\left(v_{1}, v_{2}\right) C_{t}\left(v_{1}, v_{2}\right) d v_{1} d v_{2} \\
& \quad=\int_{[0,1]^{2}} h\left(v_{1}, v_{2}\right) C_{1}\left(v_{1}, v_{2}\right) d v_{1} d v_{2}-\int_{0}^{t} B_{s}\left(h, C_{s}\right) d s .
\end{aligned}
$$

Proof.

We fix a test function $h$ from $H^{1}\left(\mathbb{R}^{2}\right)$. First to "bound" the support, we set $h_{b}\left(v_{1}, v_{2}\right)=h\left(v_{1}, v_{2}\right) \Psi\left(v_{1}\right) \Psi\left(v_{2}\right)$, where $\Psi$ is an auxiliary function defined by formula (65). Let $h_{k}, k=1,2, \ldots$, be a sequence of $C_{0}^{\infty}$ functions with which approximate $h_{b}\left(v_{1}, v_{2}\right)$ in Sobolev norm $\|\cdot\|_{H^{1}}$.

Theorem 6.1 implies that for every $k$

$$
\int_{[0,1]^{2}} h_{k}\left(v_{1}, v_{2}\right) C_{t}\left(v_{1}, v_{2}\right) d v_{1} d v_{2}
$$




$$
=\int_{[0,1]^{2}} h_{k}\left(v_{1}, v_{2}\right) C_{1}\left(v_{1}, v_{2}\right) d v_{1} d v_{2}-\int_{0}^{t} B_{s}\left(h_{k}, C_{s}\right) d s .
$$

All three terms converge as $k \rightarrow \infty$. Indeed:

for all $t \geq 0$

$$
\begin{aligned}
& \left|\int_{[0,1]^{2}} h_{k}\left(v_{1}, v_{2}\right) C_{t}\left(v_{1}, v_{2}\right) d v_{1} d v_{2}-\int_{[0,1]^{2}} h\left(v_{1}, v_{2}\right) C_{t}\left(v_{1}, v_{2}\right) d v_{1} d v_{2}\right| \\
& =\left|\int_{[0,1]^{2}} h_{k}\left(v_{1}, v_{2}\right) C_{t}\left(v_{1}, v_{2}\right) d v_{1} d v_{2}-\int_{[0,1]^{2}} h_{b}\left(v_{1}, v_{2}\right) C_{t}\left(v_{1}, v_{2}\right) d v_{1} d v_{2}\right| \\
& =\left|\int_{[0,1]^{2}}\left(h_{k}\left(v_{1}, v_{2}\right)-h_{b}\left(v_{1}, v_{2}\right)\right) C_{t}\left(v_{1}, v_{2}\right) d v_{1} d v_{2}\right| \\
& \leq \int_{[0,1]^{2}}\left|h_{k}\left(v_{1}, v_{2}\right)-h_{b}\left(v_{1}, v_{2}\right)\right| d v_{1} d v_{2} \\
& \leq \quad\left\|h_{k}-h_{b}\right\|_{L^{2}} \leq\left\|h_{k}-h_{b}\right\|_{H^{1}} \rightarrow 0 .
\end{aligned}
$$

Similarly, basing on Lemma 6.4 with $T=t$ we get

$$
\begin{aligned}
& \left|\int_{0}^{t} B_{s}\left(h_{k}, C_{s}\right) d s-\int_{0}^{t} B_{s}\left(h, C_{s}\right) d s\right| \\
& \quad=\left|\int_{0}^{t} B_{s}\left(h_{k}, C_{s}\right) d s-\int_{0}^{t} B_{s}\left(h_{b}, C_{s}\right) d s\right| \\
& \quad=\left|\int_{0}^{t} B_{s}\left(h_{k}-h_{b}, C_{s}\right) d s\right| \\
& \quad \leq \int_{0}^{t}\left|B_{s}\left(h_{k}-h_{b}, C_{s}\right)\right| d s \\
& \quad \leq t \cdot L_{t}|| h_{k}-h_{b} \|_{H^{1}} \rightarrow 0 .
\end{aligned}
$$

Lemma 6.5. Let the test function $h$ belong to $H^{1}\left(\mathbb{R}^{2}\right)$, then the function

$$
B_{h}(t)=B_{t}\left(h, C_{t}\right):[0, \infty] \longrightarrow \mathbb{R}
$$

is continuous.

Proof.

Step 1. Let $h \in C_{c}^{\infty}$.

We recall formula (52).

$$
B_{h}(t)=-E\left(\sum_{(i, j) \in I} \frac{\partial^{i+j} H}{\partial u_{1}^{i} \partial u_{2}^{j}}\left(U_{1, t}, U_{2, t}\right) L_{i, j}\left(t, U_{1, t}, U_{2, t}\right)\right),
$$

where

$$
L_{1,0}=v_{1,1}, \quad L_{0,1}=v_{2,1}, \quad L_{2,0}=\frac{1}{2} v_{1,2}^{2}, \quad L_{0,2}=\frac{1}{2} v_{2,2}^{2}, \quad L_{1,1}=v_{1,2} v_{2,2} A_{C}
$$


By assumption the processes $X_{t}^{1}$ and $X_{t}^{2}$ are path-continuous. Since their distribution functions $F_{j}(t, x)$ are continuous, the random variables

$$
U_{j, t}=F_{j}\left(t, X_{t}^{j}\right)
$$

are path-continuous as well. Now, since the convolutions $H$ are continuously differentiable and the coefficients $L_{i, j}$ are continuous, the composed stochastic process

$$
L_{t}(H)\left(U_{1, t}, U_{2, t}\right)=\sum_{(i, j) \in I} \frac{\partial^{i+j} H}{\partial u_{1}^{i} \partial u_{2}^{j}}\left(U_{1, t}, U_{2, t}\right) L_{i, j}\left(t, U_{1, t}, U_{2, t}\right)
$$

is path-continuous. Since it is bounded, we apply the Lebesgue dominated convergence theorem

$$
\begin{aligned}
& \lim _{s \rightarrow t} E\left(L_{t}(H)\left(U_{1, t}, U_{2, t}\right)-L_{s}(H)\left(U_{1, s}, U_{2, s}\right)\right) \\
& =E\left(\lim _{s \rightarrow t}\left(L_{t}(H)\left(U_{1, t}, U_{2, t}\right)-L_{s}(H)\left(U_{1, s}, U_{2, s}\right)\right)\right)=E(0)=0 .
\end{aligned}
$$

Which implies that $B_{h}(t)$ is continuous at each $t \geq 0$.

Step 2. The general case $h \in H^{1}\left(\mathbb{R}^{2}\right)$.

Let $h_{k}, k=1,2, \ldots$, be a sequence of $C^{\infty}$ functions with compact supports which approximate $h_{b}\left(v_{1}, v_{2}\right)=h\left(v_{1}, v_{2}\right) \cdot \min \left(1,\left(5-\left(v_{1}^{2}+v_{2}^{2}\right)\right)^{+}\right)$in Sobolev norm $\|\cdot\|_{H^{1}}($ compare [9] §5.3).

Since for any $t \geq 0 \quad B_{t}(h, C)=B_{t}\left(h_{b}, C\right)$ we get that the functions $B_{h_{k}}(t)$ converge almost uniformly to $B_{h}(t)$. Indeed, we fix $T>1$, then

$$
\begin{aligned}
\sup \left(\left|B_{h}(t)-B_{h_{k}}(t)\right|: t \in[1, T]\right) & =\sup \left(\left|B_{h_{b}}(t)-B_{h_{k}}(t)\right|: t \in[1, T]\right) \\
& =\sup \left(\left|B_{t}\left(h_{b}-h_{k}, C_{t}\right)\right|: t \in[1, T]\right) \\
& \leq M|| h_{b}-\left.h_{k}\right|_{H^{1}} \rightarrow 0
\end{aligned}
$$

Almost uniform convergence implies the continuity.

To conclude the proofs of the theorems, one has to observe that Corollary 6.1 and Lemma 6.5 imply Theorem 4.1, and the same Corollary and Lemma 6.4 imply Theorem 4.2.

\subsection{Comonotonicity}

The study of the form $B_{t}$ simplifies for singular copulas with support reduced to a union of segments. Especially for the copula

$$
M\left(v_{1}, v_{2}\right)=\min \left(v_{1}, v_{2}\right),
$$

having support equal to the diagonal section of the unit square. Note that the weak derivatives of $M$ are discontinuous.

$$
D_{1}(M)\left(v_{1}, v_{2}\right) \stackrel{a s}{=} \kappa\left(v_{1}-v_{2}\right), \quad D_{2}(M)\left(v_{1}, v_{2}\right) \stackrel{a s}{=} \kappa\left(v_{2}-v_{1}\right) .
$$

Lemma 6.6. For all $t \in[0,+\infty)$ and $h \in H^{1}\left(\mathbb{R}^{2}\right)$

$$
B_{t}(h, M)=\frac{1}{2} \int_{0}^{1} h(v, v)\left(v_{1,2}(t, v)^{2}+v_{2,2}(1, v)^{2}+2 v_{1,2}(t, v) v_{2,2}(1, v) A_{C}(t, v, v)\right) d v \text {. }
$$

Proof.

Applying (81), we obtain:

$$
B_{t}(h, M)=\frac{1}{2} \int_{0}^{1} \int_{0}^{1} D_{1}\left(h\left(v_{1}, v_{2}\right) v_{1,2}\left(t, v_{1}\right)^{2}\right) \kappa\left(v_{1}-v_{2}\right) d v_{1} d v_{2}
$$




$$
\begin{aligned}
& +\frac{1}{2} \int_{0}^{1} \int_{0}^{1} D_{2}\left(h\left(v_{1}, v_{2}\right) v_{2,2}\left(t, v_{2}\right)^{2}\right) \kappa\left(v_{2}-v_{1}\right) d v_{2} d v_{1} \\
& +\int_{0}^{1} \int_{0}^{1} D_{2}\left(h\left(v_{1}, v_{2}\right) v_{1,2}\left(t, v_{1}\right) v_{2,2}\left(t, v_{2}\right) A_{C}\left(t, v_{1}, v_{2}\right)\right) \kappa\left(v_{1}-v_{2}\right) d v_{2} d v_{1} \\
& =\frac{1}{2} \int_{0}^{1} \int_{0}^{v_{2}} D_{1}\left(h\left(v_{1}, v_{2}\right) v_{1,2}\left(t, v_{1}\right)^{2}\right) d v_{1} d v_{2} \\
& +\frac{1}{2} \int_{0}^{1} \int_{0}^{v_{1}} D_{2}\left(h\left(v_{1}, v_{2}\right) v_{2,2}\left(t, v_{2}\right)^{2}\right) d v_{2} d v_{1} \\
& +\int_{0}^{1} \int_{v_{1}}^{1} D_{2}\left(h\left(v_{1}, v_{2}\right) v_{1,2}\left(t, v_{1}\right) v_{2,2}\left(t, v_{2}\right) A_{C}\left(t, v_{1}, v_{2}\right)\right) d v_{2} d v_{1} .
\end{aligned}
$$

Since the functions $v_{j, 2}$ vanish at $v_{j}=0$ and at $v_{j}=1$, we get:

$$
\begin{aligned}
B_{t}(h, M) & =\frac{1}{2} \int_{0}^{1} h\left(v_{2}, v_{2}\right) v_{1,2}\left(t, v_{2}\right)^{2} d v_{2} \\
& +\frac{1}{2} \int_{0}^{1} h\left(v_{1}, v_{1}\right) v_{2,2}\left(t, v_{1}\right)^{2} d v_{1} \\
& -\int_{0}^{1} h\left(v_{1}, v_{1}\right) v_{1,2}\left(t, v_{1}\right) v_{2,2}\left(t, v_{1}\right) A_{C}\left(t, v_{1}, v_{1}\right) d v_{1} \\
& =\frac{1}{2} \int_{0}^{1} h(v, v)\left(v_{1,2}(t, v)^{2}+v_{2,2}(t, v)^{2}-2 v_{1,2}(t, v) v_{2,2}(t, v) A_{C}(t, v, v)\right) d v
\end{aligned}
$$

Note that, since $\left|A_{C}\left(t, v_{1}, v_{2}\right)\right|$ is bounded by 1 (see $\mathbf{A 3}$ ) the quadratic form

$$
R(t, v)=v_{1,2}(t, v)^{2}+v_{2,2}(t, v)^{2}-2 v_{1,2}(t, v) v_{2,2}(t, v) A_{C}(t, v, v)
$$

is nonnegative. Furthermore it vanishes only when

$$
A_{C}(t, v, v)=1, \quad v_{1,2}(t, v)=v_{2,2}(t, v) \text {, }
$$

or

$$
A_{C}(t, v, v)=-1, \quad v_{1,2}(t, v)=-v_{2,2}(t, v) \text {, }
$$

or

$$
v_{1,2}(t, v)=v_{2,2}(t, v)=0 .
$$

The above conditions (84), (85) and (86) are equivalent to

$$
\left|v_{1,2}(t, v)\right|=\left|v_{2,2}(t, v)\right| \quad \text { and } \quad v_{1,2}(t, v)=v_{2,2}(t, v) A_{C}(t, v, v) \text {. }
$$

We recall that the Spearman $\rho$ is given by a formula

$$
\rho(C)=12 \int_{[0,1]^{2}} C\left(v_{1}, c_{2}\right) d v_{1} d v_{2}-3 .
$$


the range of $\rho$ is $[-1,1]$ and the maximum is attained only at $C=M$.

We apply Theorem 4.1 for a test function $h$ equal 1 on the unit square

$$
\forall\left(v_{1}, v_{2}\right) \in[0,1]^{2} \quad h\left(v_{1}, v_{2}\right)=1
$$

We get

$$
\frac{d}{d t} \rho\left(C_{t}\right)=-12 B_{t}\left(h, C_{t}\right)
$$

Now if for $t \in[0, T] C_{t}=M$ then $\rho\left(C_{t}\right)=1$ and $B_{t}(h, M)=B_{t}\left(h, C_{t}\right)=0$. Hence for $t \in[0, T]$ and $v \in[0,1]$ condition (87) must occur. This concludes the proof of Proposition 4.1.

\subsection{Semigroups}

In this section we provide proofs of Theorems 5.1 and 5.2, i.e. we show how to construct the bivariate stochastic processes $\left(X^{1, s, C}, X^{2, s, C}\right)$ and the generator of the semigroup $\Lambda$.

\section{Proof of Theorem 5.1.}

Let $C$ be a bivariate copula and $s$ a nonnegative real number. It is well-known that for a bivariate distribution function $F\left(x_{1}, x_{2}\right)=C\left(F_{1}\left(s, x_{1}\right), F_{2}\left(s, x_{2}\right)\right)$ there exists a pair of random variables $\left(Y^{1, s, C}, Y^{2, s, C}\right)$ defined on a probability space $\left(\Omega^{s, C}, \mathcal{M}^{s, C}, \mathbb{P}^{s, C}\right)$ such, that their distribution function equals $F$. Next let $\tilde{W}=\left(\tilde{W}^{1}, \tilde{W}^{2}\right)$ be a standard two-dimensional Wiener process defined on the probability space $\left(\Omega^{W}, \mathcal{M}^{W}, \mathbb{P}^{W}\right)$ and let $\mathcal{F}^{W}$ denotes the natural filtration of $\tilde{W}$.

We extend the random variables $\left(Y^{1, s, C}, Y^{2, s, C}\right)$ and the Wiener process $\tilde{W}$ to the Cartesian product of probability spaces

$$
\left(\Omega^{W} \times \Omega^{s, C}, \sigma\left(\mathcal{M}^{W} \times \mathcal{M}^{s, C}\right), \mathbb{P}^{W} \times \mathbb{P}^{s, C}\right) .
$$

Obviously the extensions, which will be denoted by the same symbols, are independent. We extent the filtration $\mathcal{F}$ as well

$$
\mathcal{F}_{t}^{e x t}=\sigma\left\{\mathcal{V} \times \mathcal{W}: \mathcal{V} \in \mathcal{F}_{t}^{W}, \mathcal{W} \in \mathcal{M}^{s, C}\right\} .
$$

We define the bivariate stochastic process $\left(X_{t}^{1, s, C}, X_{t}^{2, s, C}\right)_{t \geq 0}$ as a $\mathcal{F}^{\text {ext }}$ adapted path-continuous solution of the following system of stochastic differential equations:

$$
\begin{aligned}
d X_{t}^{1}= & \mu_{1}\left(t+s, X_{t}^{1}\right) d t+\sigma_{1}\left(t+s, X_{t}^{1}\right) d \tilde{W}_{t}^{1}, \\
d X_{t}^{2}= & \mu_{2}\left(t+s, X_{t}^{2}\right) d t+\sigma_{2}\left(t,+s X_{t}^{2}\right) A\left(t+s, X_{t}^{1}, X_{t}^{2}\right) d \tilde{W}_{t}^{1} \\
& +\sigma_{2}\left(t+s, X_{t}^{2}\right) B\left(t+s, X_{t}^{1}, X_{t}^{2}\right) d \tilde{W}_{t}^{2}, \\
& A^{2}+B^{2}=1,
\end{aligned}
$$

with initial values $Y^{1, s, C}, Y^{2, s, C}$.

The existence of the solution is ensured by the assumptions A2. and A5.. Indeed, $\mu_{1}, \mu_{2}, \sigma_{1}, A \sigma_{2}$ and $B \sigma_{2}$ are Lipschitz functions, hence of bounded growth and fulfill the assumptions of the theorems on the existence of the solutions of SDE (see for example [18] Thm 3.4).

Note that due to the Lévy characterization theorem the process

$$
\hat{W}_{t}=\int_{0}^{t} A\left(\xi+s, X_{\xi}^{1, s, C}, X_{\xi}^{2, s, C}\right) \tilde{W}_{\xi}^{1}+\int_{0}^{t} B\left(\xi+s, X_{\xi}^{1, s, C}, X_{\xi}^{2, s, C}\right) \tilde{W}_{\xi}^{2}
$$

is a Wiener one (compare [1] Prop. 5.58). Thus $X^{2, s, C}$ fulfills the equation

$$
d X_{t}^{2, s, C}=\mu_{2}\left(t+s, X_{t}^{2, s, C}\right) d t+\sigma_{2}\left(t,+s X_{t}^{2, s, C}\right) d \hat{W}_{t} .
$$


Furthermore

$$
\begin{aligned}
d\left\langle X^{1, s, C}, X^{2, s, C}\right\rangle_{t} & =\sigma_{1}\left(t, X_{t}^{1, s, C}\right) \sigma_{2}\left(t, X_{t}^{2, s, C}\right) d\left\langle\tilde{W}^{1}, \hat{W}\right\rangle_{t} \\
& =\sigma_{1}\left(t, X_{t}^{1, s, C}\right) \sigma_{2}\left(t, X_{t}^{2, s, C}\right) A\left(t, X_{t}^{1, s, C}, X_{t}^{2, s, C}\right) d t
\end{aligned}
$$

The above concludes the proof of the first part of Theorem 5.1. Indeed the pair $X^{1, s, C}, X^{2, s, C}$ fulfills conditions A1. - A4. with shifted parameters.

The uniqueness follows from the fact that every pair of stochastic processes $\left(X^{1}, X^{2}\right)$ which fulfill A1.-A6. with Wiener processes $\left(W^{1}, W^{2}\right)$ is a solution of SDE (89) with Wiener processes $\left(\tilde{W}^{1}, \tilde{W}^{2}\right)$

$$
\tilde{W}_{t}^{1}=W_{t}^{1}, \quad d \tilde{W}_{t}^{2}=\frac{1}{B\left(t, X_{t}^{1}, X_{t}^{2}\right)} d W_{t}^{2}-\frac{A\left(t, X_{t}^{1}, X_{t}^{2}\right)}{B\left(t, X_{t}^{1}, X_{t}^{2}\right)} d W_{t}^{1}, \quad \tilde{W}_{0}^{2}=0 .
$$

Note that, since the Wiener processes defined by (94) are quadratically uncorrelated, due to the Lévy characterization theorem they form together a bivariate Wiener process (see [21] Theorem 8.6.1).

Let us fix $s \geq 0$ and $C \in \mathcal{C}^{2}$. Since weak solutions of SDE (89) are law equivalent (see [4]), all the possible two-dimensional processes $\left(X^{1, s, C}, X^{2, s, C}\right)$ are law equivalent as well.

Proof of Theorem 5.2.

We have

$$
\begin{aligned}
\frac{(i d, j) \circ \Lambda_{t}(s, C)-(s, j(C))}{t}-\left(1,-B_{s, C}\right) & =\frac{\left(s+t, j\left(C_{t}^{s, C}\right)\right)-(s, j(C))}{t}-\left(1,-B_{s, C}\right) \\
& =\left(0, \frac{j\left(C_{t}^{s, C}\right)-j(C)}{t}+B_{s, C}\right) .
\end{aligned}
$$

In order to determine the limit in the ${ }^{\star}$ weak topology, we evaluate the above with test functions from $H^{1}\left(\mathbb{R}^{2}\right)$. From Theorem 4.1, for any $h \in H^{1}\left(\mathbb{R}^{2}\right)$ we get

$$
\begin{aligned}
& \lim _{t \rightarrow 0^{+}}\left(\frac{j\left(C_{t}^{s, C}\right)-j(C)}{t}+B_{s, C}\right)(h) \\
= & \lim _{t \rightarrow 0^{+}} \frac{1}{t}\left(\int_{[0,1]^{2}} h\left(v_{1}, v_{2}\right) C_{t}^{s, C}\left(v_{1}, v_{2}\right) d v_{1} d v_{2}-\int_{[0,1]^{2}} h\left(v_{1}, v_{2}\right) C\left(v_{1}, v_{2}\right) d v_{1} d v_{2}\right)+B_{s}(h, C) \\
= & \left.\frac{d}{d t} \int_{[0,1]^{2}} h\left(v_{1}, v_{2}\right) C_{t}^{s, C}\left(v_{1}, v_{2}\right) d v_{1} d v_{2}\right|_{t=0}+B_{S}\left(h, C_{0}^{s, C}\right)=0 .
\end{aligned}
$$

Hence in the * weak topology on $H^{1}\left(\mathbb{R}^{2}\right)^{\star}$

$$
\lim _{t \rightarrow 0^{+}} \frac{(i d, j) \circ \Lambda_{t}(s, C)-(s, j(C))}{t}-\left(1,-B_{s, C}\right)=0 .
$$

Generator $L$ is defined on the whole state space $\mathbb{R}^{+} \times \mathcal{C}^{2}$ and $L(s, C)$ equals $\left(1,-B_{s, C}\right)$.

\section{References}

[1] Baudoin, F. (2014). Diffusion Processes and Stochastic Calculus. European Mathematical Society, Zürich. 
[2] Bibbona, E., L. Sacerdote, and E. Torre (2016). A copula-based method to build diffusion models with prescribed marginal and serial dependence. Methodol. Comput. Appl. Probab. 18(3), 765-783.

[3] Brezis, H. (2011). Functional Analysis, Sobolev Spaces and Partial Differential Equations. Springer, New York.

[4] Cherny, A. S. (2002). On the uniqueness in law and the pathwise uniqueness for stochastic differential equations. Theory Probab. Appl. 46(3), 406-419.

[5] Cherubini, U., E. Luciano, and W. Vecchiato (2004). Copula Methods in Finance. John Wiley \& Sons, Chichester.

[6] Choe, H. J., C. Ahn, B. J. Kim, and Y.-K. Ma (2013). Copulas from the Fokker-Planck equation. J. Math. Anal. Appl. 406(2), 519-530.

[7] Darsow, W. F., B. Nguyen, and E. T. Olsen (1992). Copulas and Markov processes. Illinois J. Math. 36(4), 600-642.

[8] Durante, F. and C. Sempi (2016). Principles of Copula Theory. CRC Press, Boca Raton FL.

[9] Evans, L. C. (1998). Partial Differential Equations. American Mathematical Society, Providence RI.

[10] Gihman, I. I. and A. V. Skorohod (1972). Stochastic Differential Equations. Springer, New York.

[11] Gilbarg, D. and N. S. Trudinger (2001). Elliptic Partial Differential Equations of Second Order. Second edition. Springer, Berlin.

[12] Jaworski, P. and M. Krzywda (2013). Coupling of Wiener processes by using copulas. Statist. Probab. Lett. 83(9), $2027-2033$.

[13] Jaworski, P. and M. Krzywda (2019). On copulas of self-similar Ito processes. Working paper.

[14] Jaworski, P., F. Durante, and W. Härdle, editors (2013). Copulae in Mathematical and Quantitative Finance. Springer, Heidelberg.

[15] Jaworski, P., F. Durante, W. Härdle, and T. Rychlik, editors (2010). Copula Theory and its Applications. Springer, Heidelberg.

[16] Joe, H. (1997). Multivariate Models and Dependence Concepts. Chapman \& Hall, London.

[17] Joe, H. (2015). Dependence Modeling with Copulas. CRC Press, Boca Raton FL.

[18] Khasminskii, R. (2012). Stochastic Stability of Differential Equations. Second edition. Springer, Berlin.

[19] Maz'ya, V. (2011). Sobolev Spaces. Second edition. Springer, Berlin.

[20] Nelsen, R. B. (2006). An Introduction to Copulas. Second edition. Springer, New York.

[21] Oksendal, B. (2003). Stochastic Differential Equations. Sixth edition. Springer, Berlin.

[22] Resnick, S. I. (2007). Heavy-Tail Phenomena. Springer, New York.

[23] Schmitz, V. (2003). Copulas and Stochastic Processes. Shaker, Aachen.

[24] Schuss, Z. (2010). Theory and Applications of Stochastic Processes. Springer, New York.

[25] Sempi, C. (2010). Coupled brownian motion. In C. Borgelt, G. González Rodriguez, W. Trutschnig, M. A. Lubiano, M. Ángeles Gil, P. Grzegorzewski, and O. Hryniewicz (Eds.), Combining Soft Computing and Statistical Methods in Data Analysis, pp. 569-574. Springer, Berlin.

[26] Sempi, C. (2016). Copulae of processes related to the Brownian motion: A brief survey. In S. Saminger-Platz and R. Mesiar (Eds.), On Logical, Algebraic, and Probabilistic Aspects of Fuzzy Set Theory, pp. 173-180. Springer, Basel.

[27] Trutschnig, W. (2011). On a strong metric on the space of copulas and its induced dependence measure. J. Math. Anal. Appl. 384(2), 690-705. 